\title{
New Possibilities of Hybrid Texture of Neutrino Mass Matrix
}

\author{
Madan Singh \\ Department of Physics, National Institute of Technology Kurukshetra, Haryana 136119, India \\ Correspondence should be addressed to Madan Singh; singhmadan179@gmail.com \\ Received 15 April 2018; Revised 26 May 2018; Accepted 4 June 2018; Published 11 July 2018 \\ Academic Editor: Andrzej Okniński
}

Copyright (C) 2018 Madan Singh. This is an open access article distributed under the Creative Commons Attribution License, which permits unrestricted use, distribution, and reproduction in any medium, provided the original work is properly cited. The publication of this article was funded by SCOAP ${ }^{3}$.

We investigate the novel possibilities of hybrid textures comprising a vanishing minor (or element) and two equal elements (or cofactors) in light neutrino mass matrix $M_{\gamma}$. Such type of texture structures leads to sixty phenomenological cases each, out of which only fifty-six are viable with texture containing a vanishing minor and an equality between the elements in $M_{\nu}$, while fifty are found to be viable with texture containing a vanishing element and an equality of cofactors in $M_{\gamma}$ under the current experimental test at $3 \sigma$ confidence level. Detailed numerical analysis of all the possible cases has been presented.

\section{Introduction}

During the last two decades, our knowledge regarding the neutrino sector has been enriched to a great extent, thanks to solar, atmospheric, reactor, and accelerator based experiments which convincingly reveal that neutrinos have nonzero and nondegenerate masses and can convert from one flavor to another. While the developments over the past two decades have brought out a coherent picture of neutrino mixing, there are still several intriguing issues without which our understanding of neutrino physics remains incomplete. For instance, the present available data does not throw any light on the neutrino mass spectrum, which may be normal/inverted and may even be degenerate. In addition, nature of neutrino mass whether Dirac or Majorana particle, determination of absolute neutrino mass, leptonic $\mathrm{CP}$ violation, and Dirac CP phase $\delta$ are still open issues. Also the information regarding the lightest neutrino mass has to be sharpened further to pinpoint the specific possibility of neutrino mass spectrum.

After the precise measurement of reactor mixing angle $\theta_{13}$ in T2K, MINOS, Double Chooz, Daya Bay, and RENO experiments [1-5], five parameters in the neutrino sector have been well measured by neutrino oscillation experiments. In general, there are nine parameters in the lightest neutrinos mass matrix. The remaining four unknown parameters may be taken as the lightest neutrino mass, the Dirac CP-violating phase, and two Majorana phases. The Dirac CP-violating phase is expected to be measured in future long baseline neutrino experiments, and the lightest mass can be determined from beta decay and cosmological experiments. If neutrinoless double-beta decay $(0 \nu \beta \beta)$ is detected, a combination of the two Majorana phases can also be probed. Clearly, the currently available data on neutrino masses and mixing are insufficient for an unambiguous reconstruction of neutrino mass matrices.

In the lack of a convincing fermion flavor theory, several phenomenological ansatz have been proposed in the literature as some elements of neutrino mass matrix are considered to be zero or equal [6-26] or some cofactors of neutrino mass matrix are considered to be either zero or equal $[6,27-$ 35]. The main motivation for invoking different mass matrix ansatz is to relate fermion masses and mixing angles in a testable manner which reduces the number of free parameters in the neutrino mass matrix. In particular, mass matrices with zero textures (or cofactors) have been extensively studied [1026, 29-35] due to their connections to flavor symmetries. In addition, texture specific mass matrices with one zero element (or minor) and an equality between two independent elements (or cofactors) have also been studied in the literature $[7-9,27,28]$. Out of sixty possibilities, only fifty-four are found to be compatible with the neutrino oscillation data [9] for texture structures having one zero element and an equal matrix elements in the neutrino mass matrix (also known as 
hybrid texture), while for texture with one vanishing minor and an equal cofactors in the neutrino mass matrix (also known as inverse hybrid texture) only fifty-two cases are able to survive the data $[27,28]$.

In the present paper, we propose the novel possibilities of hybrid textures where we assume one texture zero and an equality between the cofactors (referred as type $\mathrm{X}$ ) or one zero minor and an equality between the elements (referred as type $\mathrm{Y}$ ) in the Majorana neutrino mass matrix $M_{v}$. Such type of texture structures sets two conditions on the parameter space and hence reduces the number of free parameters to seven. Therefore the proposed texture structures are as predictive as texture two zeros and any other hybrid textures.

In [6], it is demonstrated that an equality between the elements of $M_{\nu}$ can be realized through type-II seesaw mechanism [36-40] while an equality between cofactors of $M_{\nu}$ can be generated from type-I seesaw mechanism [4144]. The zeros element (or minor) in $M_{\nu}$ can be obtained using $Z_{n}$ flavor symmetry $[29-35,45]$. Therefore the viable cases of proposed hybrid texture can be realized within the framework of seesaw mechanism.

In the present work, we have systematically investigated all the of sixty possible cases belonging to type $\mathrm{X}$ and type $\mathrm{Y}$ structures, respectively. We have studied the implication of these textures for Dirac CP-violating phase $(\delta)$ and two Majorana phases $(\rho, \sigma)$. We, also, calculate the effective Majorana mass and lowest neutrino mass for all viable hybrid textures belonging to type $\mathrm{X}$ and type $\mathrm{Y}$ structures. In addition, we present the correlation plots between different parameters of the hybrid textures of neutrinos for $3 \sigma$ allowed ranges of the known parameters.

The layout of the paper is planned as follows: in Section 2, we shall discuss the methodology to obtain the constraint equations. Section 3 is devoted to numerical analysis. Section 4 will summarize our result.

\section{Methodology}

Before proceeding further, we briefly underline the methodology relating the elements of the mass matrices to those of the mixing matrix. In the flavor basis, where the charged lepton mass matrix is diagonal, the Majorana neutrino mass matrix can be expressed as

$$
M_{\nu}=P_{l} U P_{\nu} M^{\text {diag }} P_{\nu}^{T} U^{T} P_{l}^{T},
$$

where $M^{\text {diag }}=\operatorname{diag}\left(m_{1}, m_{2}, m_{3}\right)$ is the diagonal matrix of neutrino masses and $U$ is the flavor mixing matrix and

$$
\begin{aligned}
P_{\nu} & =\left(\begin{array}{ccc}
e^{i \rho} & 0 & 0 \\
0 & e^{i \sigma} & 0 \\
0 & 0 & 1
\end{array}\right), \\
P_{l} & =\left(\begin{array}{ccc}
e^{i \phi_{e}} & 0 & 0 \\
0 & e^{i \phi_{\mu}} & 0 \\
0 & 0 & e^{i \phi_{\tau}}
\end{array}\right),
\end{aligned}
$$

where $P_{v}$ is diagonal phase matrix containing Majorana neutrinos $\rho, \sigma . P_{l}$ is unobservable phase matrix and depends on phase convention. Equation (1) can be rewritten as

$$
\begin{aligned}
M_{\nu} & =\left(\begin{array}{lll}
M_{e e} & M_{e \mu} & M_{e \tau} \\
M_{e \mu} & M_{\mu \mu} & M_{\mu \tau} \\
M_{e \tau} & M_{\mu \tau} & M_{\tau \tau}
\end{array}\right) \\
& =P_{l} U\left(\begin{array}{ccc}
\lambda_{1} & 0 & 0 \\
0 & \lambda_{2} & 0 \\
0 & 0 & \lambda_{3}
\end{array}\right) U^{T} P_{l}^{T},
\end{aligned}
$$

where $\lambda_{1}=m_{1} e^{2 i \rho}, \lambda_{2}=m_{2} e^{2 i \sigma}$, and $\lambda_{3}=m_{3}$. For the present analysis, we consider the following parameterization of $U[46]$ :

$U$

$$
=\left(\begin{array}{ccc}
c_{12} c_{13} & s_{12} c_{13} & s_{13} \\
-c_{12} s_{23} s_{13}-s_{12} c_{23} e^{-i \delta} & -s_{12} s_{23} s_{13}+c_{12} c_{23} e^{-i \delta} & s_{23} c_{13} \\
-c_{12} c_{23} s_{13}+s_{12} s_{23} e^{-i \delta} & -s_{12} c_{23} s_{13}-c_{12} s_{23} e^{-i \delta} & c_{23} c_{13}
\end{array}\right),
$$

where $c_{i j}=\cos \theta_{i j}$ and $s_{i j}=\sin \theta_{i j}$. Here, $U$ is a $3 \times 3$ unitary matrix consisting of three flavor mixing angles $\left(\theta_{12}, \theta_{23}, \theta_{13}\right)$ and one Dirac CP-violating phase $\delta$.

For the illustration of type $\mathrm{X}$ and $\mathrm{Y}$ structures, we consider a case $A_{1}$, satisfying following conditions:

$$
C_{11}=M_{\mu \mu} M_{\tau \tau}-M_{\mu \tau} M_{\mu \tau}=0
$$

and

$$
M_{e \mu}-M_{e \tau}=0,
$$

for type $\mathrm{X}$, while in case of type $\mathrm{Y}$, it contains

$$
M_{e e}=0
$$

and

$$
C_{12}-C_{13}=0 \text {, }
$$

or

$$
\begin{aligned}
& (-1) \cdot\left(M_{e \tau} M_{\tau \tau}-M_{\mu \tau} M_{e \tau}\right)-\left(M_{e \mu} M_{\mu \tau}-M_{\mu \mu} M_{e \tau}\right) \\
& \quad=0
\end{aligned}
$$

where $C_{i j}$ denotes cofactor corresponding to $i^{\text {th }}$ row and $j^{\text {th }}$ column. Then $A_{1}$ can be denoted in a matrix form as

$$
\left(\begin{array}{ccc}
0 & \Delta & \Delta \\
\Delta & \times & \times \\
\Delta & \times & \times
\end{array}\right)
$$

where " $\Delta$ " stands for nonzero and equal elements (or cofactors), while "0" stands for vanishing element (or minor) in neutrino mass matrix. " $x$ " stands for arbitrary elements. 
2.1. One Vanishing Minor with Two Equal Elements of $M_{v}$. Using (1), any element $M_{p q}$ in the neutrino mass matrix can be expressed in terms of mixing matrix elements as

$$
M_{p q}=e^{i\left(\phi_{p}+\phi_{q}\right)} \sum_{i=1,2,3} U_{p i} U_{q i} \lambda_{i}
$$

where $p, q$ run over $\mathrm{e}, \mu$, and $\tau$, and $e^{i\left(\phi_{p}+\phi_{q}\right)}$ is phase factor.

The existence of a zero minor in the Majorana neutrino mass matrix implies

$$
M_{p q} M_{r s}-M_{t u} M_{v w}=0
$$

The above condition yields a complex equation as

$$
\begin{aligned}
& \sum_{i, j=1,2,3}\left(e^{i\left(\phi_{p}+\phi_{q}+\phi_{r}+\phi_{s}\right)} U_{p i} U_{q i} U_{r j} U_{s j}\right. \\
& \left.\quad-e^{i\left(\phi_{t}+\phi_{u}+\phi_{v}+\phi_{w}\right)} U_{t i} U_{u i} U_{v j} U_{w j}\right) \lambda_{i} \lambda_{j}=0,
\end{aligned}
$$

It is observed that for any cofactor there is an inherent property as $\phi_{p}+\phi_{q}+\phi_{r}+\phi_{s}=\phi_{t}+\phi_{u}+\phi_{v}+\phi_{w}$. Thus we can extract this total phase factor from the bracket in (13).

Hence (13) can be rewritten as

$$
X_{3} \lambda_{1} \lambda_{2}+X_{1} \lambda_{2} \lambda_{3}+X_{2} \lambda_{3} \lambda_{1}=0
$$

where

$$
X_{k}=\left(U_{p i} U_{q i} U_{r j} U_{s j}-U_{t i} U_{u i} U_{v j} U_{w j}\right)+(i \longleftrightarrow j),
$$

with $(i, j, k)$ as the cyclic permutation of $(1,2,3)$.

On the other hand, the condition of two equal elements in $M_{v}$ yields following:

$$
M_{a b}-M_{c d}=0
$$

Equation (16) yields a following complex equation:

$$
\sum_{i=1,2,3}\left(P_{1} U_{a i} U_{b i}-P_{2} U_{c i} U_{d i}\right) \lambda_{i}=0
$$

where $P_{1}=e^{i\left(\phi_{a}+\phi_{b}\right)}$ and $P_{2}=e^{i\left(\phi_{c}+\phi_{d}\right)}$.

Or

$$
\sum_{i=1,2,3}\left(P U_{a i} U_{b i}-U_{c i} U_{d i}\right) \lambda_{i}=0
$$

where $P \equiv P_{1} / P_{2}=e^{i(a+b-c-d)}$ and $a, b, c$, and $d$ run over $e, \mu$, and $\tau$.

Equation (18) can be rewritten as

$$
Y_{1} \lambda_{1}+Y_{2} \lambda_{2}+Y_{3} \lambda_{3}=0
$$

where $Y_{1}=\left(P U_{a 1} U_{b 1}-U_{c 1} U_{d 1}\right), Y_{2}=\left(P U_{a 2} U_{b 2}-U_{c 2} U_{d 2}\right)$, and $Y_{3}=\left(P U_{a 3} U_{b 3}-U_{c 3} U_{d 3}\right)$.

Solving (14) and (19) simultaneously leads to the following complex mass ratio in terms of $\left(\lambda_{13}\right)_{ \pm}$:

$$
\left(\lambda_{13}\right)_{+}=\frac{-\left(Y_{1} X_{1}-Y_{2} X_{2}+Y_{3} X_{3}+\sqrt{C}\right)}{2 Y_{1} X_{3}},
$$

and

$$
\left(\lambda_{13}\right)_{-}=\frac{-\left(Y_{1} X_{1}-Y_{2} X_{2}+Y_{3} X_{3}-\sqrt{C}\right)}{2 Y_{1} X_{3}} .
$$

Using (14), (20), and (21), we obtain the relations for complex mass ratio in terms of $\left(\lambda_{23}\right)_{ \pm}$

$$
\left(\lambda_{23}\right)_{+}=-\frac{X_{2}}{X_{3}} \times \frac{Y_{1} X_{1}-Y_{2} X_{2}+Y_{3} X_{3}+\sqrt{C}}{-Y_{1} X_{1}-Y_{2} X_{2}+Y_{3} X_{3}+\sqrt{C}},
$$

and

$$
\left(\lambda_{23}\right)_{-}=-\frac{X_{2}}{X_{3}} \times \frac{Y_{1} X_{1}-Y_{2} X_{2}+Y_{3} X_{3}-\sqrt{C}}{-Y_{1} X_{1}-Y_{2} X_{2}+Y_{3} X_{3}-\sqrt{C}},
$$

where $C=\left(-Y_{1} X_{1}+Y_{2} X_{2}+Y_{3} X_{3}\right)^{2}-4 X_{2} X_{3} Y_{2} Y_{3}$ and $\left(\lambda_{13}\right)_{ \pm} \equiv$ $\left(\lambda_{1} / \lambda_{3}\right)_{ \pm}$and $\left(\lambda_{23}\right)_{ \pm} \equiv\left(\lambda_{2} / \lambda_{3}\right)_{ \pm}$. The magnitudes of the two neutrino mass ratios in (20), (21), (22), and (23) are given by $\xi_{ \pm}=\left|\left(\lambda_{13}\right)_{ \pm}\right|$and $\zeta_{ \pm}=\left|\left(\lambda_{23}\right)_{ \pm}\right|$, while the Majorana CPviolating phases $\rho$ and $\sigma$ can be given as $\rho=(1 / 2) \arg \left(\lambda_{13}\right)_{ \pm}$, $\sigma=(1 / 2) \arg \left(\lambda_{23}\right)_{ \pm}$.

2.2. One Vanishing Element with Two Equal Cofactors of $M_{\gamma}$. If one of the elements of $M_{\nu}$ is considered zero [e.g., $M_{\alpha \beta}=0$ ], we obtain the following constraint equation:

$$
\sum_{i=1,2,3} U_{\alpha i} U_{\beta i} \lambda_{i}=0
$$

or

$$
\lambda_{1} A_{1}+\lambda_{2} A_{2}+\lambda_{3} A_{3}=0,
$$

where $A_{1}=U_{\alpha 1} U_{\beta 1}, A_{2}=U_{\alpha 2} U_{\beta 2}$, and $A_{3}=U_{\alpha 3} U_{\beta 3}$.

The condition for two equal cofactors [e.g., $C_{m n}=C_{m^{\prime} n^{\prime}}$ ] in neutrino mass matrix implies

$$
\begin{aligned}
& (-1)^{m+n}\left(M_{a b} M_{c d}-M_{e f} M_{g h}\right) \\
& \quad-(-1)^{m^{\prime}+n^{\prime}}\left(M_{a^{\prime} b^{\prime}} M_{c^{\prime} d^{\prime}}-M_{e^{\prime} f^{\prime}} M_{g^{\prime} h^{\prime}}\right)=0,
\end{aligned}
$$

or

$$
\begin{aligned}
& \sum_{i, j=1,2,3}\left\{(-1)^{m+n}\left(Q_{3} U_{a i} U_{b i} U_{c j} U_{d j}-Q_{4} U_{e i} U_{f i} U_{g j} U_{h j}\right)\right. \\
& -(-1)^{m^{\prime}+n^{\prime}} \\
& \left.\cdot\left(Q_{5} U_{a^{\prime} i} U_{b^{\prime} i} U_{c^{\prime} j} U_{d^{\prime} j}-Q_{6} U_{e^{\prime} i} U_{f^{\prime} i} U_{g^{\prime} j} U_{h^{\prime} j}\right)\right\} \lambda_{i} \lambda_{j} \\
& =0,
\end{aligned}
$$

where $Q_{3}=Q_{4}$ and $Q_{5}=Q_{6}$ due to inherent property of any cofactor. Thus we can write

$$
\begin{aligned}
& \sum_{i, j=1,2,3}\left\{(-1)^{m+n} Q_{3}\left(U_{a i} U_{b i} U_{c j} U_{d j}-U_{e i} U_{f i} U_{g j} U_{h j}\right)\right. \\
& -(-1)^{m^{\prime}+n^{\prime}} \\
& \left.\cdot Q_{5}\left(U_{a^{\prime} i} U_{b^{\prime} i} U_{c^{\prime} j} U_{d^{\prime} j}-U_{e^{\prime} i} U_{f^{\prime} i} U_{g^{\prime} j} U_{h^{\prime} j}\right)\right\} \lambda_{i} \lambda_{j} \\
& =0,
\end{aligned}
$$


or

$$
\begin{aligned}
& \sum_{i, j=1,2,3}\left\{(-1)^{m+n} Q\left(U_{a i} U_{b i} U_{c j} U_{d j}-U_{e i} U_{f i} U_{g j} U_{h j}\right)\right. \\
& \left.-(-1)^{m^{\prime}+n^{\prime}}\left(U_{a^{\prime} i} U_{b^{\prime} i} U_{c^{\prime} j} U_{d^{\prime} j}-U_{e^{\prime} i} U_{f^{\prime} i} U_{g^{\prime} j} U_{h^{\prime} j}\right)\right\} \\
& \cdot \lambda_{i} \lambda_{j}=0,
\end{aligned}
$$

where $Q \equiv Q_{3} / Q_{5}=e^{i\left(\phi_{a}+\phi_{b}+\phi_{c}+\phi_{d}-\phi_{a^{\prime}}-\phi_{b^{\prime}}-\phi_{c^{\prime}}-\phi_{d^{\prime}}\right)}$.

Equation (29) can be rewritten as

$$
\lambda_{1} \lambda_{2} B_{3}+\lambda_{2} \lambda_{3} B_{1}+\lambda_{3} \lambda_{1} B_{2}=0
$$

where

$$
\begin{aligned}
B_{k} & \\
= & (-1)^{m+n} Q\left(U_{a i} U_{b i} U_{c j} U_{d j}-U_{e i} U_{f i} U_{g j} U_{h j}\right), \\
& -(-1)^{m^{\prime}+n^{\prime}}\left(U_{a^{\prime} i} U_{b^{\prime} i} U_{c^{\prime} j} U_{d^{\prime} j}-U_{e^{\prime} i} U_{f^{\prime} i} U_{g^{\prime} j} U_{h^{\prime} j}\right) \\
& +(i \longleftrightarrow j),
\end{aligned}
$$

with $(i, j, k)$ a cyclic permutation of $(1,2,3)$.

Solving (25) and (30) simultaneously we obtain the analytical expressions of $\left(\lambda_{13}\right)_{ \pm}$

$$
\left(\lambda_{13}\right)_{+}=\frac{-\left(B_{1} A_{1}-B_{2} A_{2}+B_{3} A_{3}+\sqrt{D}\right)}{2 B_{1} A_{3}},
$$

and

$$
\left(\lambda_{13}\right)_{-}=\frac{-\left(B_{1} A_{1}-B_{2} A_{2}+B_{3} A_{3}-\sqrt{D}\right)}{2 B_{1} A_{3}} .
$$

Using (30), (32), and (33), we get the relations for complex mass ratio in terms of $\left(\lambda_{23}\right)_{ \pm}$

$$
\left(\lambda_{23}\right)_{+}=-\frac{B_{2}}{B_{3}} \times \frac{\left(B_{1} A_{1}-B_{2} A_{2}+B_{3} A_{3}+\sqrt{D}\right)}{\left(-B_{1} A_{1}-B_{2} A_{2}+B_{3} A_{3}+\sqrt{D}\right)},
$$

and

$$
\left(\lambda_{23}\right)_{-}=-\frac{B_{2}}{B_{3}} \times \frac{\left(B_{1} A_{1}-B_{2} A_{2}+B_{3} A_{3}-\sqrt{D}\right)}{\left(-B_{1} A_{1}-B_{2} A_{2}+B_{3} A_{3}-\sqrt{D}\right)},
$$

where $D=\left(-B_{1} A_{1}+B_{2} A_{2}+B_{3} A_{3}\right)^{2}-4 A_{2} A_{3} B_{2} B_{3}$.

The magnitudes of the two neutrino mass ratios are given by $\xi_{ \pm}=\left|\left(\lambda_{13}\right)_{ \pm}\right|$and $\zeta_{ \pm}=\left|\left(\lambda_{23}\right)_{ \pm}\right|$, while the Majorana CPviolating phases $\rho$ and $\sigma$ can be given as $\rho=(1 / 2) \arg \left(\lambda_{13}\right)_{ \pm}$, $\sigma=(1 / 2) \arg \left(\lambda_{23}\right)_{ \pm}$.

The solar and atmospheric mass-squared differences $\left(\delta m^{2}, \Delta m^{2}\right)$, where $\delta m^{2}$ corresponds to solar mass-squared difference and $\Delta m^{2}$ corresponds to atmospheric masssquared difference, can be defined as [8]

$$
\begin{aligned}
\delta m^{2} & =\left(m_{2}^{2}-m_{1}^{2}\right), \\
\Delta m^{2} & =m_{3}^{2}-\frac{1}{2}\left(m_{1}^{2}+m_{2}^{2}\right) .
\end{aligned}
$$

The sign of $\Delta m^{2}$ is still unknown: $\Delta m^{2}>0$ or $\Delta m^{2}<0$ implies normal mass spectrum (NS) or inverted mass spectrum (IS). The lowest neutrino mass $\left(m_{0}\right)$ is $m_{1}$ for NS and $m_{3}$ for IS. The experimentally determined solar and atmospheric neutrino mass-squared differences can be related to $\xi$ and $\zeta$ as

$$
R_{v} \equiv \frac{\delta m^{2}}{\left|\Delta m^{2}\right|}=\frac{2\left(\zeta^{2}-\xi^{2}\right)}{\left|2-\left(\zeta^{2}+\xi^{2}\right)\right|},
$$

and the three neutrino masses can be determined using following relations:

$$
\begin{aligned}
& m_{3}=\sqrt{\frac{\delta m^{2}}{\zeta^{2}-\xi^{2}}}, \\
& m_{2}=m_{3} \zeta \\
& m_{1}=m_{3} \xi .
\end{aligned}
$$

From the analysis, it is found that cases belonging to type X (or type Y) exhibit the identical phenomenological implications and are related through permutation symmetry $[36-40,46]$. This corresponds to permutation of the 2-3 rows and 2-3 columns of $M_{v}$. The corresponding permutation matrix can be given by

$$
P_{23}=\left(\begin{array}{lll}
1 & 0 & 0 \\
0 & 0 & 1 \\
0 & 1 & 0
\end{array}\right) .
$$

With the help of permutation symmetry, one obtains the following relations among the neutrino oscillation parameters:

$$
\begin{aligned}
& \theta_{12}^{l}=\theta_{12}^{m}, \\
& \theta_{23}^{l}=90^{\circ}-\theta_{23}^{m}, \\
& \theta_{13}^{l}=\theta_{13}^{m}, \\
& \delta^{l}=\delta^{m}-180^{\circ},
\end{aligned}
$$

where $l$ and $m$ denote the cases related by 2-3 permutation. The following pairs among sixty possibilities of type $\mathrm{X}$ (or type $\mathrm{Y}$ ) are related via permutation symmetry:

$$
\begin{aligned}
& \left(A_{1}, A_{1}\right) ; \\
& \left(A_{2}, A_{8}\right) ; \\
& \left(A_{3}, A_{7}\right) ; \\
& \left(A_{4}, A_{6}\right) ; \\
& \left(A_{5}, A_{5}\right) ; \\
& \left(A_{9}, A_{10}\right) ; \\
& \left(B_{1}, C_{1}\right) ; \\
& \left(B_{2}, C_{7}\right) ; \\
& \left(B_{3}, C_{6}\right) ;
\end{aligned}
$$




$$
\begin{aligned}
& \left(B_{4}, C_{5}\right) ; \\
& \left(B_{5}, C_{4}\right) ; \\
& \left(B_{6}, C_{3}\right) \\
& \left(B_{7}, C_{2}\right) ; \\
& \left(B_{8}, C_{10}\right) ; \\
& \left(B_{9}, C_{9}\right) ; \\
& \left(B_{10}, C_{8}\right) ; \\
& \left(D_{1}, F_{2}\right) ; \\
& \left(D_{2}, F_{1}\right) ; \\
& \left(D_{3}, F_{4}\right) ; \\
& \left(D_{4}, F_{3}\right) ; \\
& \left(D_{5}, F_{5}\right) ; \\
& \left(D_{6}, F_{9}\right) ; \\
& \left(D_{7}, F_{8}\right) ; \\
& \left(D_{8}, F_{7}\right) ; \\
& \left(D_{9}, F_{6}\right) ; \\
& \left(D_{10}, F_{10}\right) ; \\
& \left(E_{1}, E_{2}\right) ; \\
& \left(E_{3}, E_{4}\right) ; \\
& \left(E_{5}, E_{5}\right) ; \\
& \left(E_{6}, E_{9}\right) ; \\
& \left(E_{7}, E_{8}\right) ; \\
& \left(E_{10}, E_{10}\right) \\
&
\end{aligned}
$$

Clearly we are left with only thirty-two independent cases. It is worthwhile mentioning that $A_{1}, A_{5}, E_{5}$, and $E_{10}$ are invariant under the permutations of 2 - and 3 -rows and columns.

\section{Numerical Analysis}

The experimental constraints on neutrino parameters at $3 \sigma$ confidence level (CL) are given in Table 1. The effective Majorana mass relevant for neutrinoless double-beta $(0 \nu \beta \beta)$ decay is given by

$$
|M|_{e e}=\left|m_{1} c_{12}^{2} c_{13}^{2} e^{2 i \rho}+m_{2} s_{12}^{2} c_{13}^{2} e^{2 i \sigma}+m_{3} s_{13}^{2}\right| .
$$

This effective mass is just the absolute value of $M_{e e}$ component of the neutrino mass matrix. The observation of $0 \nu \beta \beta$
TABLE 1: Current neutrino oscillation parameters from global fits at $3 \sigma$ confidence level (CL) $[47,48]$. NS(IS) refers to normal (inverted) neutrino mass spectrum.

\begin{tabular}{lcc}
\hline Parameter & Best Fit & $3 \sigma$ \\
\hline$\delta m^{2}\left[10^{-5} \mathrm{eV}^{2}\right]$ & 7.50 & $7.03-8.09$ \\
\hline$\left|\Delta m_{31}^{2}\right|\left[10^{-3} \mathrm{eV}^{2}\right]$ (NS) & 2.52 & $2.407-2.643$ \\
\hline$\left|\Delta m_{31}^{2}\right|\left[10^{-3} \mathrm{eV}^{2}\right]$ (IS) & 2.52 & $2.39-2.63$ \\
\hline$\theta_{12}$ & $33.56^{\circ}$ & $31.3^{\circ}-35.99^{\circ}$ \\
\hline$\theta_{23}$ (NS) & $41.6^{\circ}$ & $38.4^{\circ}-52.8^{\circ}$ \\
\hline$\theta_{23}$ (IS) & $50.0^{\circ}$ & $38.8^{\circ}-53.1^{\circ}$ \\
\hline$\theta_{13}$ (NS) & $8.46^{\circ}$ & $7.99^{\circ}-8.90^{\circ}$ \\
\hline$\theta_{13}$ (IS) & $8.49^{\circ}$ & $8.03^{\circ}-8.93^{\circ}$ \\
\hline$\delta$ (NS) & $261^{\circ}$ & $0^{\circ}-360^{\circ}$ \\
\hline$\delta$ (IS) & $277^{\circ}$ & $145^{\circ}-391^{\circ}$ \\
\hline
\end{tabular}

would establish neutrinos to be Majorana particles. Data from KamLAND-Zen experiment has presented an improved search for neutrinoless double-beta $(0 \nu \beta \beta)$ decay [49-51] and it is found that $|M|_{e e}<(0.061-0.165) e V$ at $90 \%($ or $<2 \sigma) \mathrm{CL}$. For recent reviews on $0 \nu \beta \beta$ decay, see [49-51].

In the present analysis, we consider more conservative upper bound on $|M|_{e e}$, i.e., $|M|_{e e}<0.5 e V$ at $3 \sigma$ CL [52]. We span the parameter space of input neutrino oscillation parameters $\left(\theta_{12}, \theta_{23}, \theta_{13}, \Delta m^{2}, \Delta m^{2}\right)$ lying in their $3 \sigma$ ranges by randomly generating points of the order of $10^{7}$. Since the Dirac CP-violating phase $\delta$ is experimentally unconstrained at $3 \sigma$ level, therefore, we vary $\delta$ within its full possible range $\left[0^{\circ}, 360^{\circ}\right]$. Using (38) and the experimental inputs on neutrino mixing angles and mass-squared differences, the parameter space of $\delta, \rho, \sigma$ and $|M|_{e e}$, and $m_{0}$ can be subsequently constrained.

In Figures 1, 2, 3, 4, 5, 6, 7, 8, 9, 10, 11, and 12 we demonstrate the correlations for $A_{1}, B_{2}, D_{7}$, and $E_{1}$ cases. Since there are large numbers of viable cases, therefore it is not practically possible to show all the plots. We have simply taken arbitrary independent cases from each category for the purpose of illustration of our results. The predictions regarding three $\mathrm{CP}$ violating phases $(\rho, \sigma, \delta)$, effective neutrino mass $|M|_{e e}$, and lowest neutrino mass $m_{o}$ for all the allowed cases of type X and type $\mathrm{Y}$ textures have been encapsulated in Tables 3, 4, 5 , and 6 . Before proceeding further, it is worth pointing out that the phenomenological results for $\rho, \sigma, \delta,|M|_{e e}$, and $m_{o}$ have been obtained using the two possible solutions of $\lambda_{13}$ and $\lambda_{23}$, respectively [ (20), (21), (22), and (23)]. All the sixty phenomenologically possible cases belonging to type $\mathrm{X}$ and type $\mathrm{Y}$ texture structures have been divided into six categories A, B, C, D, E, and F (Table 2). Among them a large number of cases are found to overlap in their predictions regarding $\delta$, $\rho, \sigma|M|_{e e}$, and $m_{0}$ and are related via permutation symmetry as pointed out earlier. The main results and the discussion are summarized as follows.

Category $A$. In Category $\mathrm{A}$, all the ten cases $A_{1}, A_{2}, A_{3}$, $A_{4}, A_{5}, A_{6}, A_{7}, A_{8}, A_{9}$, and $A_{10}$ are found to be viable with the data at $3 \sigma \mathrm{CL}$ for type $\mathrm{X}$ structure, and normal mass spectrum (NS) remainS ruled out for all these cases (Table 3 ). 


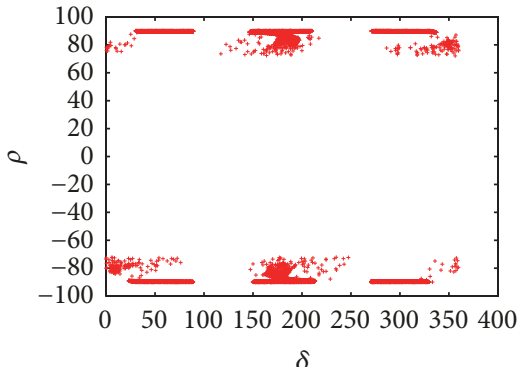

(a)

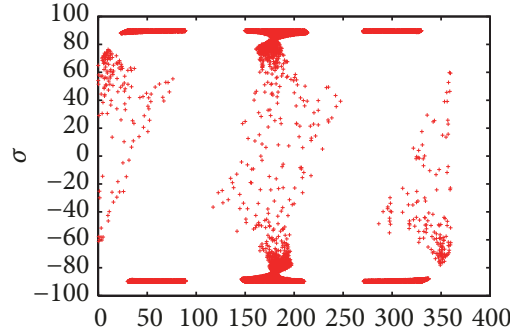

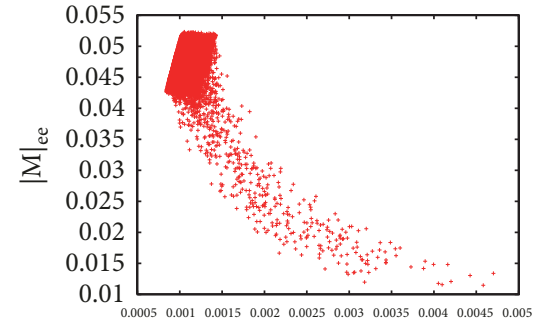

$\mathrm{m}_{\mathrm{o}}$

(c)

Figure 1: Correlation plots for texture $A_{1}$ (IS) for type X at $3 \sigma$ CL. The symbols have their usual meaning. $\delta, \rho$, and $\sigma$ are measured in degrees, while $|M|_{e e}$ and $m_{0}$ are in $\mathrm{eV}$ units.

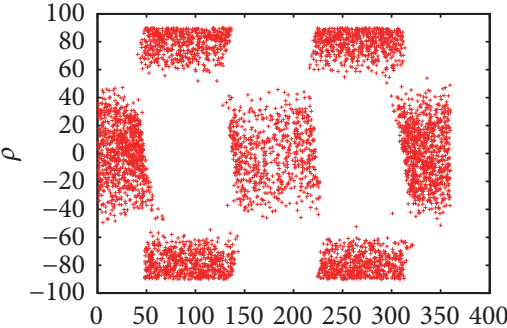

$\delta$

(a)

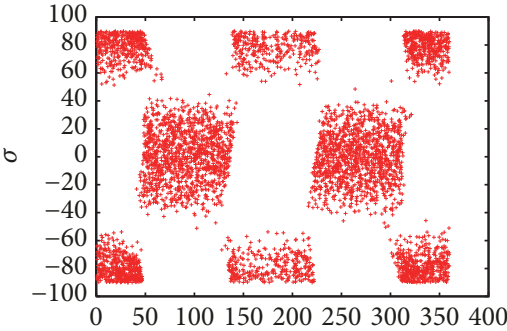

$\delta$

(b)

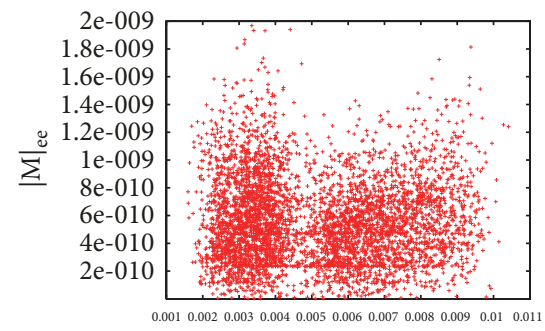

$\mathrm{m}_{\mathrm{o}}$

(c)

Figure 2: Correlation plots for texture $A_{1}$ (NS) for type Y at $3 \sigma$ CL. The symbols have their usual meaning. $\delta, \rho$, and $\sigma$ are measured in degrees, while $|M|_{e e}$ and $m_{0}$ are in $\mathrm{eV}$ units.

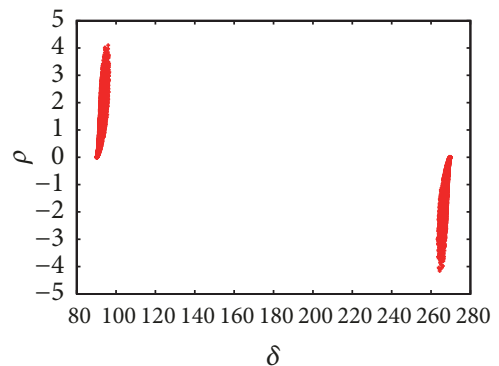

(a)

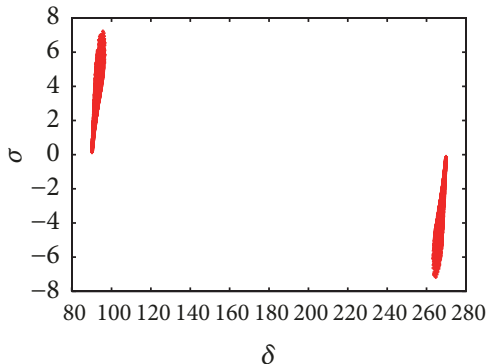

(b)

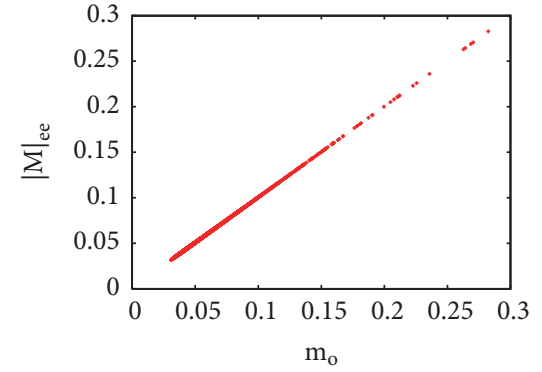

(c)

FIgure 3: Correlation plots for texture $B_{2}$ (NS) for type X at $3 \sigma$ CL. The symbols have their usual meaning. $\delta, \rho$, and $\sigma$ are measured in degrees, while $|M|_{e e}$ and $m_{0}$ are in $\mathrm{eV}$ units.

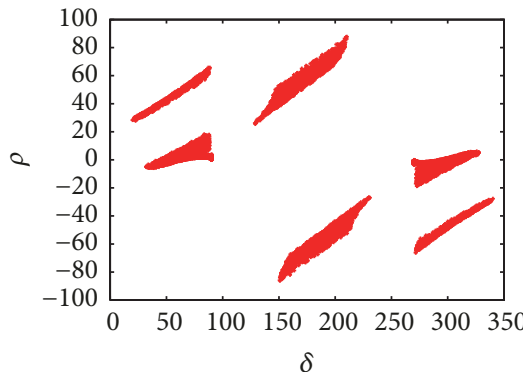

(a)

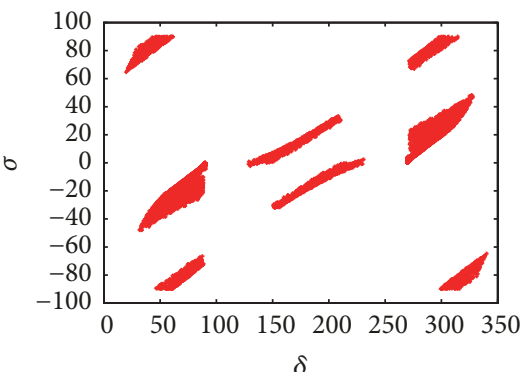

(b)

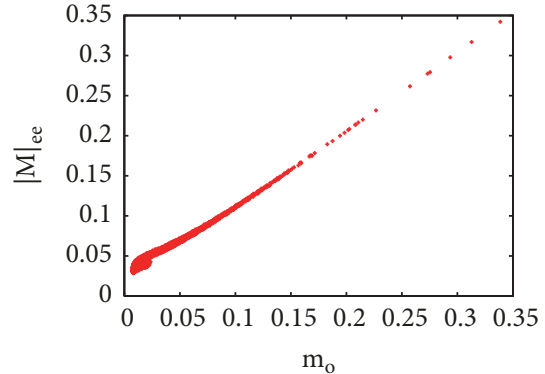

(c)

Figure 4: Correlation plots for texture $B_{2}$ (IS) for type $\mathrm{X}$ at $3 \sigma \mathrm{CL}$. The symbols have their usual meaning. $\delta$, $\rho$, and $\sigma$ are measured in degrees, while $|M|_{e e}$ and $m_{0}$ are in $\mathrm{eV}$ units. 


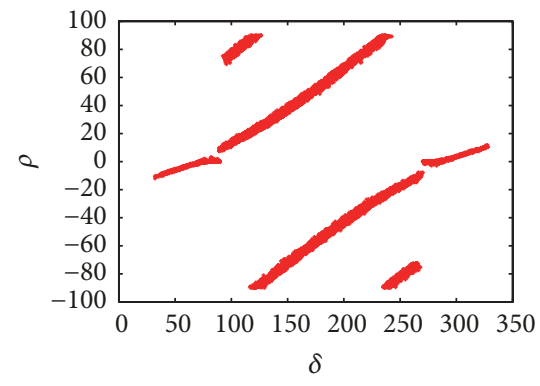

(a)

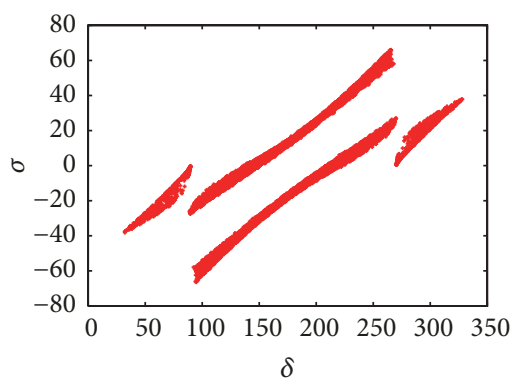

(b)

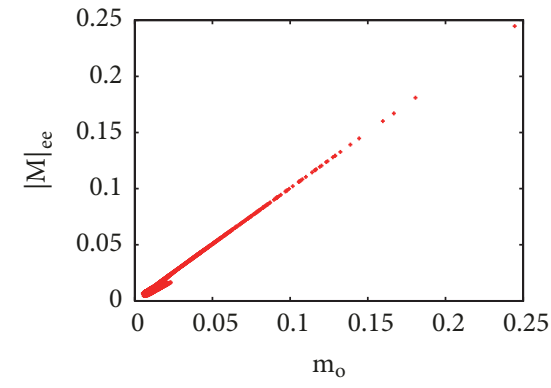

(c)

Figure 5: Correlation plots for texture $B_{2}$ (NS) for type Y at $3 \sigma$ CL. The symbols have their usual meaning. $\delta, \rho$, and $\sigma$ are measured in degrees, while $|M|_{e e}$ and $m_{0}$ are in $\mathrm{eV}$ units.

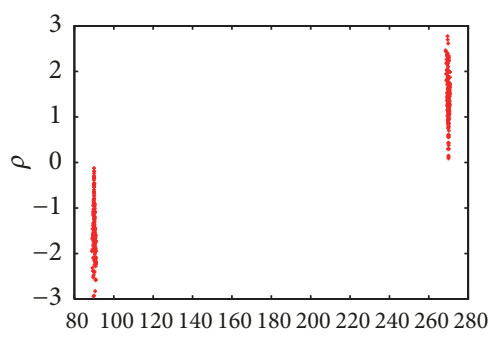

$\delta$

(a)

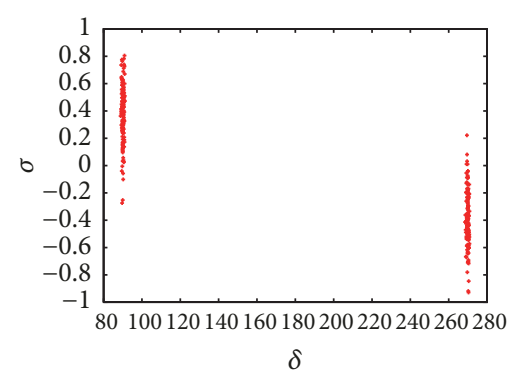

(b)

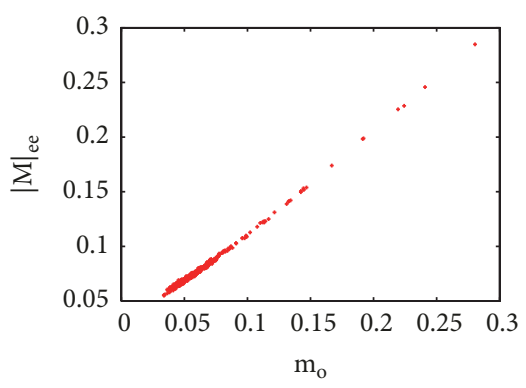

(c)

FiguRE 6: Correlation plots for texture $B_{2}$ (IS) for type Y at $3 \sigma$ CL. The symbols have their usual meaning. $\delta, \rho$, and $\sigma$ are measured in degrees, while $|M|_{e e}$ and $m_{0}$ are in $\mathrm{eV}$ units.

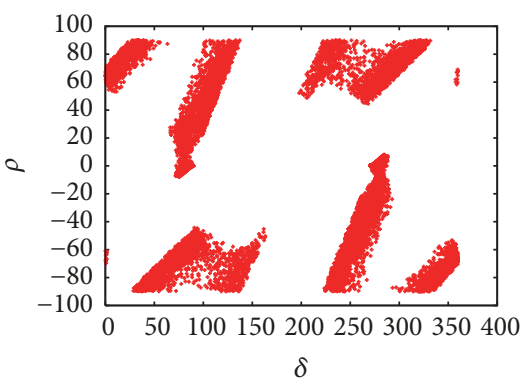

(a)

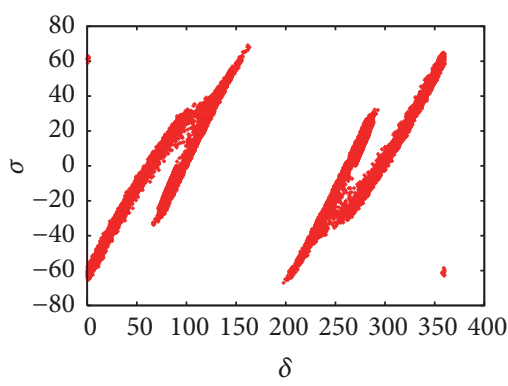

(b)

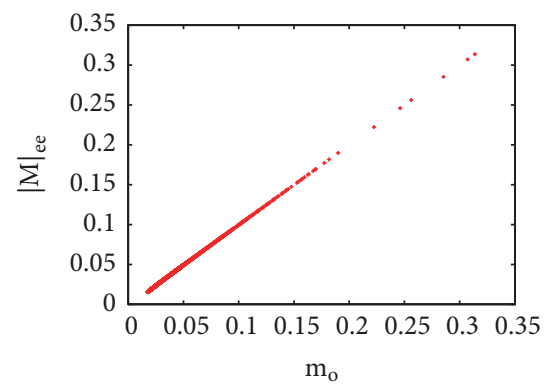

(c)

Figure 7: Correlation plots for texture $D_{7}$ (NS) for type X at $3 \sigma$ CL. The symbols have their usual meaning. $\delta, \rho$, and $\sigma$ are measured in degrees, while $|M|_{e e}$ and $m_{0}$ are in $\mathrm{eV}$ units.

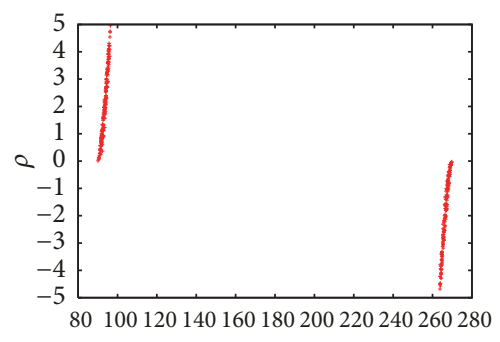

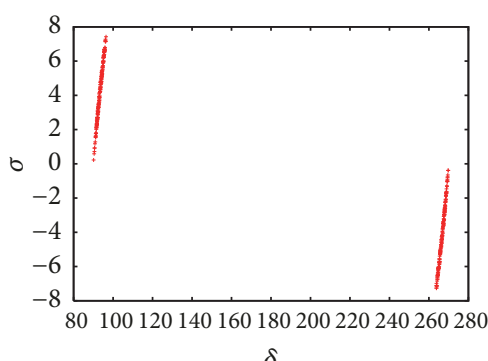

(b)

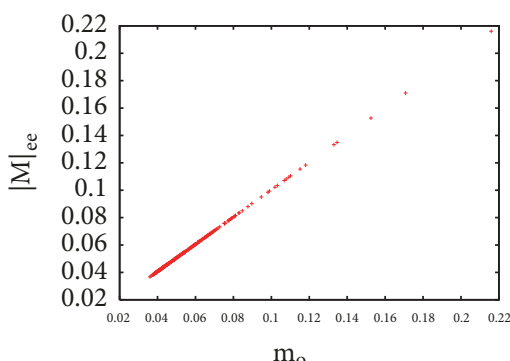

(c)

FIGURE 8: Correlation plots for texture $D_{7}$ (IS) for type $\mathrm{X}$ at $3 \sigma \mathrm{CL}$. The symbols have their usual meaning. $\delta$, $\rho$, and $\sigma$ are measured in degrees, while $|M|_{e e}$ and $m_{0}$ are in $\mathrm{eV}$ units. 


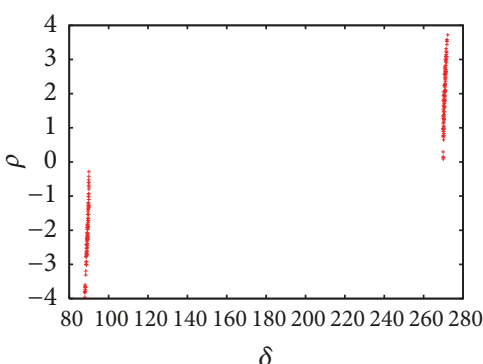

(a)

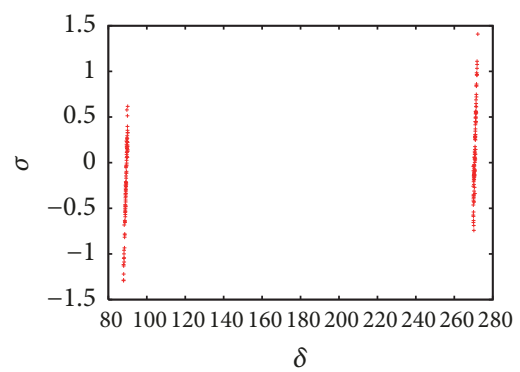

(b)

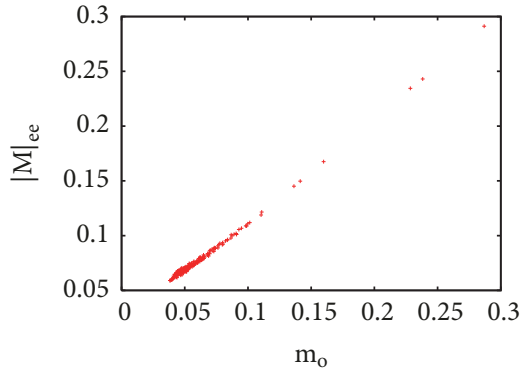

(c)

FIgURE 9: Correlation plots for texture $D_{7}$ (NS) for type Y at $3 \sigma$ CL. The symbols have their usual meaning. $\delta, \rho$, and $\sigma$ are measured in degrees, while $|M|_{e e}$ and $m_{0}$ are in $\mathrm{eV}$ units.

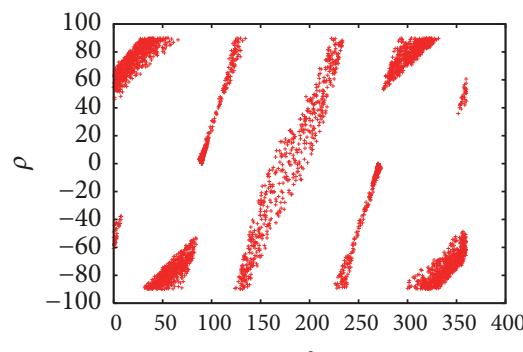

$\delta$

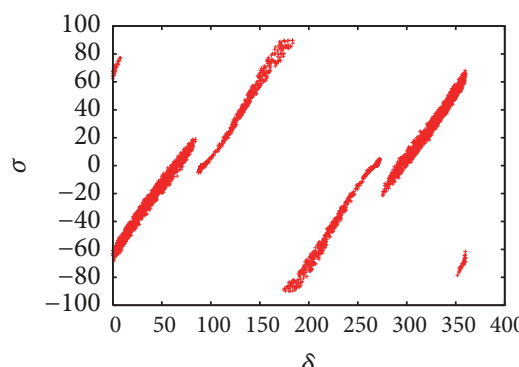

(b)

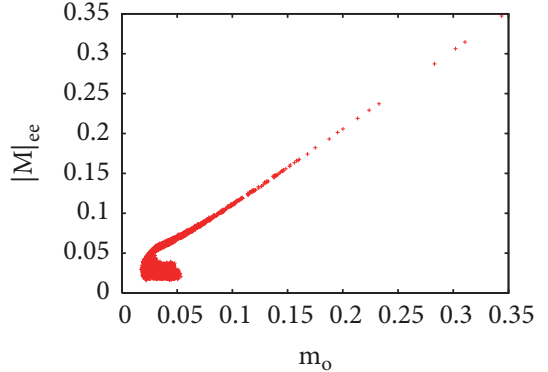

(c)

Figure 10: Correlation plots for texture $D_{7}$ (IS) for type Y at $3 \sigma$ CL. The symbols have their usual meaning. $\delta$, $\rho$, and $\sigma$ are measured in degrees, while $|M|_{e e}$ and $m_{0}$ are in $\mathrm{eV}$ units.

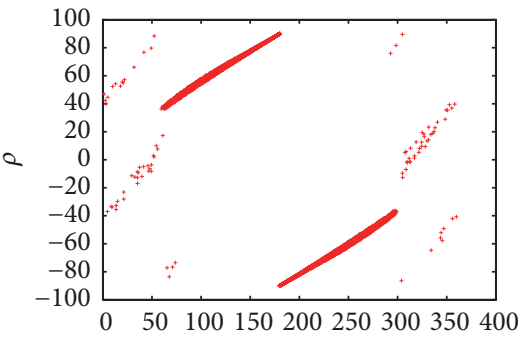

(a)

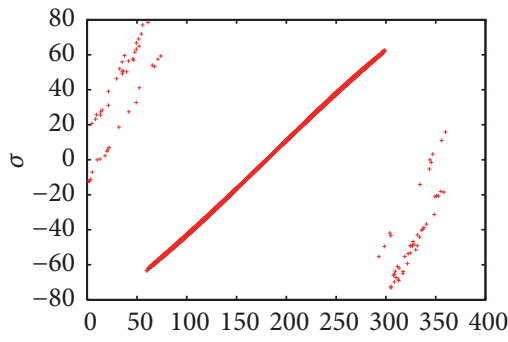

(b)

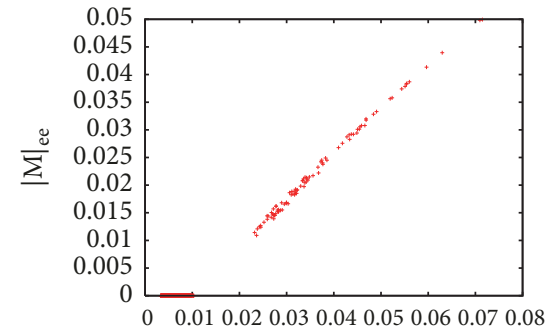

$\mathrm{m}_{\mathrm{o}}$

(c)

FIgURE 11: Correlation plots for texture $E_{1}$ (NS) for type X at $3 \sigma$ CL. The symbols have their usual meaning. $\delta$, $\rho$, and $\sigma$ are measured in degrees, while $|M|_{e e}$ and $m_{0}$ are in $\mathrm{eV}$ units.

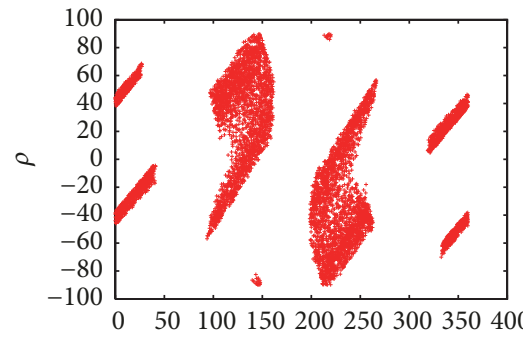

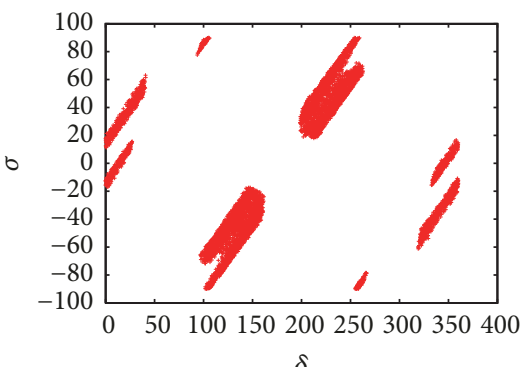

(b)

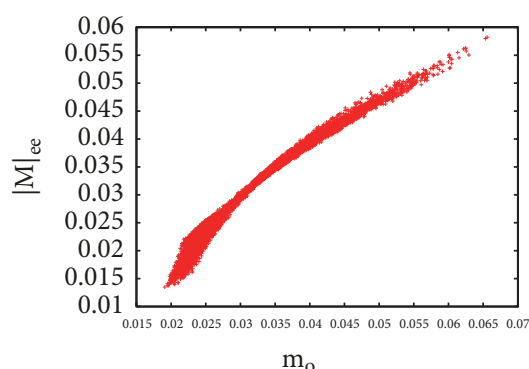

(c)

Figure 12: Correlation plots for texture $E_{1}$ (IS) for type $\mathrm{Y}$ at $3 \sigma \mathrm{CL}$. The symbols have their usual meaning. $\delta$, $\rho$, and $\sigma$ are measured in degrees, while $|M|_{e e}$ and $m_{0}$ are in $\mathrm{eV}$ units. 
TABLE 2: All the sixty phenomenological possible cases belonging to types $\mathrm{X}$ and $\mathrm{Y}$, respectively, have been shown. P and Q are unobservable phases associated with types X and Y, respectively.

\begin{tabular}{|c|c|c|c|c|}
\hline Cases & $\mathrm{X}$ & $\mathrm{P}$ & $\mathrm{Y}$ & Q \\
\hline$A_{1}$ & $C_{11}=0, M_{12}=M_{13}$ & $e^{i\left(\phi_{\mu}-\phi_{\tau}\right)}$ & $M_{11}=0, C_{12}=C_{13}$ & $e^{i\left(\phi_{\tau}-\phi_{\mu}\right)}$ \\
\hline$A_{2}$ & $C_{11}=0, M_{12}=M_{22}$ & $e^{i\left(\phi_{e}-\phi_{\mu}\right)}$ & $M_{11}=0, C_{12}=C_{22}$ & $e^{i\left(\phi_{\mu}-\phi_{e}\right)}$ \\
\hline$A_{3}$ & $C_{11}=0, M_{13}=M_{23}$ & $e^{i\left(\phi_{e}-\phi_{\mu}\right)}$ & $M_{11}=0, C_{13}=C_{23}$ & $e^{i\left(\phi_{\mu}-\phi_{e}\right)}$ \\
\hline$A_{4}$ & $C_{11}=0, M_{22}=M_{23}$ & $e^{i\left(\phi_{\mu}-\phi_{\tau}\right)}$ & $M_{11}=0, C_{22}=C_{23}$ & $e^{i\left(\phi_{\tau}-\phi_{\mu}\right)}$ \\
\hline$A_{5}$ & $C_{11}=0, M_{22}=M_{33}$ & $e^{2 i\left(\phi_{\mu}-\phi_{\tau}\right)}$ & $M_{11}=0, C_{22}=C_{33}$ & $e^{2 i\left(\phi_{\tau}-\phi_{\mu}\right)}$ \\
\hline$A_{6}$ & $C_{11}=0, M_{23}=M_{33}$ & $e^{i\left(\phi_{\mu}-\phi_{\tau}\right)}$ & $M_{11}=0, C_{23}=C_{33}$ & $e^{i\left(\phi_{\tau}-\phi_{\mu}\right)}$ \\
\hline$A_{7}$ & $C_{11}=0, M_{12}=M_{23}$ & $e^{i\left(\phi_{e}-\phi_{\tau}\right)}$ & $M_{11}=0, C_{12}=C_{23}$ & $e^{i\left(\phi_{\tau}-\phi_{e}\right)}$ \\
\hline$A_{8}$ & $C_{11}=0, M_{13}=M_{33}$ & $e^{i\left(\phi_{e}-\phi_{\tau}\right)}$ & $M_{11}=0, C_{13}=C_{33}$ & $e^{i\left(\phi_{\tau}-\phi_{e}\right)}$ \\
\hline$A_{9}$ & $C_{11}=0, M_{13}=M_{22}$ & $e^{i\left(\phi_{e}+\phi_{\tau}-2 \phi_{\mu}\right)}$ & $M_{11}=0, C_{13}=C_{22}$ & $e^{i\left(-\phi_{e}-\phi_{\tau}+2 \phi_{\mu}\right)}$ \\
\hline$A_{10}$ & $C_{11}=0, M_{12}=M_{33}$ & $e^{i\left(\phi_{e}+\phi_{\mu}-2 \phi_{\tau}\right)}$ & $M_{11}=0, C_{12}=C_{33}$ & $e^{i\left(-\phi_{e}-\phi_{\mu}+2 \phi_{\tau}\right)}$ \\
\hline$B_{1}$ & $C_{12}=0, M_{11}=M_{13}$ & $e^{i\left(\phi_{e}-\phi_{\tau}\right)}$ & $M_{12}=0, C_{11}=C_{13}$ & $e^{i\left(\phi_{\tau}-\phi_{e}\right)}$ \\
\hline$B_{2}$ & $C_{12}=0, M_{12}=M_{22}$ & $e^{i\left(\phi_{e}+\phi_{\tau}-2 \phi_{\mu}\right)}$ & $M_{12}=0, C_{12}=C_{22}$ & $e^{i\left(2 \phi_{\mu}-\phi_{e}-\phi_{\tau}\right)}$ \\
\hline$B_{3}$ & $C_{12}=0, M_{13}=M_{23}$ & $e^{i\left(\phi_{e}-\phi_{\mu}\right)}$ & $M_{12}=0, C_{13}=C_{23}$ & $e^{i\left(\phi_{\mu}-\phi_{e}\right)}$ \\
\hline$B_{4}$ & $C_{12}=0, M_{13}=M_{33}$ & $e^{i\left(\phi_{e}-\phi_{\tau}\right)}$ & $M_{12}=0, C_{13}=C_{33}$ & $e^{i\left(\phi_{\tau}-\phi_{e}\right)}$ \\
\hline$B_{5}$ & $C_{12}=0, M_{11}=M_{22}$ & $e^{2 i\left(\phi_{e}-\phi_{\mu}\right)}$ & $M_{12}=0, C_{11}=C_{22}$ & $e^{2 i\left(\phi_{\mu}-\phi_{e}\right)}$ \\
\hline$B_{6}$ & $C_{12}=0, M_{11}=M_{23}$ & $e^{i\left(2 \phi_{e}-\phi_{\mu}-\phi_{\tau}\right)}$ & $M_{12}=0, C_{11}=C_{23}$ & $e^{i\left(-2 \phi_{e}+\phi_{\mu}+\phi_{\tau}\right)}$ \\
\hline$B_{7}$ & $C_{12}=0, M_{11}=M_{33}$ & $e^{2 i\left(\phi_{e}-\phi_{\tau}\right)}$ & $M_{12}=0, C_{11}=C_{33}$ & $e^{2 i\left(\phi_{\tau}-\phi_{e}\right)}$ \\
\hline$B_{8}$ & $C_{12}=0, M_{22}=M_{23}$ & $e^{i\left(\phi_{\mu}-\phi_{\tau}\right)}$ & $M_{12}=0, C_{22}=C_{23}$ & $e^{i\left(\phi_{\tau}-\phi_{\mu}\right)}$ \\
\hline$B_{9}$ & $C_{12}=0, M_{22}=M_{33}$ & $e^{2 i\left(\phi_{\mu}-\phi_{\tau}\right)}$ & $M_{12}=0, C_{22}=C_{33}$ & $e^{2 i\left(\phi_{\tau}-\phi_{\mu}\right)}$ \\
\hline$B_{10}$ & $C_{12}=0, M_{23}=M_{33}$ & $e^{i\left(\phi_{\mu}-\phi_{\tau}\right)}$ & $M_{12}=0, C_{23}=C_{33}$ & $e^{i\left(\phi_{\tau}-\phi_{\mu}\right)}$ \\
\hline$C_{1}$ & $C_{13}=0, \quad M_{11}=M_{12}$ & $e^{i\left(\phi_{e}-\phi_{\mu}\right)}$ & $M_{13}=0, C_{11}=C_{12}$ & $e^{i\left(\phi_{\mu}-\phi_{e}\right)}$ \\
\hline$C_{2}$ & $C_{13}=0, M_{11}=M_{22}$ & $e^{2 i\left(\phi_{e}-\phi_{\mu}\right)}$ & $M_{13}=0, C_{11}=C_{22}$ & $e^{2 i\left(\phi_{\mu}-\phi_{e}\right)}$ \\
\hline$C_{3}$ & $C_{13}=0, M_{11}=M_{23}$ & $e^{i\left(2 \phi_{e}-\phi_{\mu}-\phi_{\tau}\right)}$ & $M_{13}=0, C_{11}=C_{23}$ & $e^{i\left(-2 \phi_{e}+\phi_{\mu}+\phi_{\tau}\right)}$ \\
\hline $\mathrm{C}_{4}$ & $C_{13}=0, M_{11}=M_{33}$ & $e^{2 i\left(\phi_{e}-\phi_{\tau}\right)}$ & $M_{13}=0, C_{11}=C_{33}$ & $e^{2 i\left(\phi_{\tau}-\phi_{e}\right)}$ \\
\hline$C_{5}$ & $C_{13}=0, M_{12}=M_{22}$ & $e^{i\left(\phi_{e}-\phi_{\mu}\right)}$ & $M_{13}=0, C_{12}=C_{22}$ & $e^{i\left(\phi_{\mu}-\phi_{e}\right)}$ \\
\hline $\mathrm{C}_{6}$ & $C_{13}=0, M_{12}=M_{23}$ & $e^{i\left(\phi_{e}-\phi_{\tau}\right)}$ & $M_{13}=0, C_{12}=C_{23}$ & $e^{i\left(\phi_{\tau}-\phi_{e}\right)}$ \\
\hline$C_{7}$ & $C_{13}=0, M_{12}=M_{33}$ & $e^{i\left(\phi_{e}-\phi_{\mu}-2 \phi_{\tau}\right)}$ & $M_{13}=0, C_{12}=C_{33}$ & $e^{i\left(-\phi_{e}+\phi_{\mu}+2 \phi_{\tau}\right)}$ \\
\hline $\mathrm{C}_{8}$ & $C_{13}=0, M_{22}=M_{23}$ & $e^{i\left(\phi_{\mu}-\phi_{\tau}\right)}$ & $M_{13}=0, C_{22}=C_{23}$ & $e^{i\left(\phi_{\tau}-\phi_{\mu}\right)}$ \\
\hline$C_{9}$ & $C_{13}=0, M_{22}=M_{33}$ & $e^{2 i\left(\phi_{\mu}-\phi_{\tau}\right)}$ & $M_{13}=0, C_{22}=C_{33}$ & $e^{2 i\left(\phi_{\tau}-\phi_{\mu}\right)}$ \\
\hline$C_{10}$ & $C_{13}=0, M_{23}=M_{33}$ & $e^{i\left(\phi_{\mu}-\phi_{\tau}\right)}$ & $M_{13}=0, C_{23}=C_{33}$ & $e^{i\left(\phi_{\tau}-\phi_{\mu}\right)}$ \\
\hline$D_{1}$ & $C_{22}=0, M_{11}=M_{12}$ & $e^{i\left(\phi_{e}-\phi_{\mu}\right)}$ & $M_{22}=0, C_{11}=C_{12}$ & $e^{i\left(\phi_{\mu}-\phi_{e}\right)}$ \\
\hline $\mathrm{D}_{2}$ & $C_{22}=0, M_{11}=M_{13}$ & $e^{i\left(\phi_{e}-\phi_{\tau}\right)}$ & $M_{22}=0, C_{11}=C_{13}$ & $e^{i\left(\phi_{\tau}-\phi_{e}\right)}$ \\
\hline$D_{3}$ & $C_{22}=0, M_{11}=M_{23}$ & $e^{i\left(2 \phi_{e}-\phi_{\mu}-\phi_{\tau}\right)}$ & $M_{22}=0, C_{11}=C_{23}$ & $e^{i\left(-2 \phi_{e}+\phi_{\mu}+\phi_{\tau}\right)}$ \\
\hline$D_{4}$ & $C_{22}=0, M_{11}=M_{33}$ & $e^{2 i\left(\phi_{e}-\phi_{\tau}\right)}$ & $M_{22}=0, C_{11}=C_{33}$ & $e^{2 i\left(\phi_{\tau}-\phi_{e}\right)}$ \\
\hline$D_{5}$ & $C_{22}=0, M_{12}=M_{13}$ & $e^{i\left(\phi_{\mu}-\phi_{\tau}\right)}$ & $M_{22}=0, C_{12}=C_{13}$ & $e^{i\left(\phi_{\tau}-\phi_{\mu}\right)}$ \\
\hline$D_{6}$ & $C_{22}=0, M_{12}=M_{23}$ & $e^{i\left(\phi_{e}-\phi_{\tau}\right)}$ & $M_{22}=0, C_{12}=C_{23}$ & $e^{i\left(\phi_{\tau}-\phi_{e}\right)}$ \\
\hline$D_{7}$ & $C_{22}=0, M_{12}=M_{33}$ & $e^{i\left(\phi_{e}+\phi_{\mu}-2 \phi_{\tau}\right)}$ & $M_{22}=0, C_{12}=C_{33}$ & $e^{i\left(-\phi_{e}-\phi_{\mu}+2 \phi_{\tau}\right)}$ \\
\hline$D_{8}$ & $C_{22}=0, M_{13}=M_{23}$ & $e^{i\left(\phi_{e}-\phi_{\mu}\right)}$ & $M_{22}=0, C_{13}=C_{23}$ & $e^{i\left(\phi_{\mu}-\phi_{e}\right)}$ \\
\hline$D_{9}$ & $C_{22}=0, M_{13}=M_{33}$ & $e^{i\left(\phi_{e}-\phi_{\tau}\right)}$ & $M_{22}=0, C_{13}=C_{33}$ & $e^{i\left(\phi_{\tau}-\phi_{e}\right)}$ \\
\hline$D_{10}$ & $C_{22}=0, M_{23}=M_{33}$ & $e^{i\left(\phi_{\mu}-\phi_{\tau}\right)}$ & $M_{22}=0, C_{23}=C_{33}$ & $e^{i\left(\phi_{\tau}-\phi_{\mu}\right)}$ \\
\hline$E_{1}$ & $C_{23}=0, M_{11}=M_{12}$ & $e^{i\left(\phi_{e}-\phi_{\mu}\right)}$ & $M_{23}=0, C_{11}=C_{12}$ & $e^{i\left(\phi_{\mu}-\phi_{e}\right)}$ \\
\hline$E_{2}$ & $C_{23}=0, M_{11}=M_{13}$ & $e^{i\left(\phi_{e}-\phi_{\tau}\right)}$ & $M_{23}=0, C_{11}=C_{13}$ & $e^{i\left(\phi_{\tau}-\phi_{e}\right)}$ \\
\hline$E_{3}$ & $C_{23}=0, M_{11}=M_{22}$ & $e^{2 i\left(\phi_{e}-\phi_{\mu}\right)}$ & $M_{23}=0, C_{11}=C_{22}$ & $e^{2 i\left(\phi_{\mu}-\phi_{e}\right)}$ \\
\hline$E_{4}$ & $C_{23}=0, M_{11}=M_{33}$ & $e^{2 i\left(\phi_{e}-\phi_{\tau}\right)}$ & $M_{23}=0, C_{11}=C_{33}$ & $e^{2 i\left(\phi_{\tau}-\phi_{e}\right)}$ \\
\hline$E_{5}$ & $C_{23}=0, M_{12}=M_{13}$ & $e^{i\left(\phi_{\mu}-\phi_{\tau}\right)}$ & $M_{23}=0, C_{12}=C_{13}$ & $e^{i\left(\phi_{\tau}-\phi_{\mu}\right)}$ \\
\hline$E_{6}$ & $C_{23}=0, M_{12}=M_{22}$ & $e^{i\left(\phi_{e}-\phi_{\mu}\right)}$ & $M_{23}=0, C_{12}=C_{22}$ & $e^{i\left(\phi_{\mu}-\phi_{e}\right)}$ \\
\hline
\end{tabular}


TABLE 2: Continued.

\begin{tabular}{|c|c|c|c|c|}
\hline Cases & X & $\mathrm{P}$ & $\mathrm{Y}$ & Q \\
\hline$E_{7}$ & $C_{23}=0, M_{13}=M_{33}$ & $e^{i\left(\phi_{e}-\phi_{\mu}-2 \phi_{\tau}\right)}$ & $M_{23}=0, C_{13}=C_{33}$ & $e^{i\left(-\phi_{e}+\phi_{\mu}+2 \phi_{\tau}\right)}$ \\
\hline$E_{8}$ & $C_{23}=0, M_{13}=M_{22}$ & $e^{i\left(\phi_{e}-\phi_{\tau}-2 \phi_{\mu}\right)}$ & $M_{23}=0, C_{13}=C_{22}$ & $e^{i\left(-\phi_{e}+\phi_{\tau}+2 \phi_{\mu}\right)}$ \\
\hline$E_{9}$ & $C_{23}=0, M_{13}=M_{33}$ & $e^{i\left(\phi_{e}-\phi_{\tau}\right)}$ & $M_{23}=0, C_{13}=C_{33}$ & $e^{i\left(\phi_{\tau}-\phi_{e}\right)}$ \\
\hline$E_{10}$ & $C_{23}=0, M_{22}=M_{33}$ & $e^{2 i\left(\phi_{\mu}-\phi_{\tau}\right)}$ & $M_{23}=0, C_{22}=C_{33}$ & $e^{2 i\left(\phi_{\tau}-\phi_{\mu}\right)}$ \\
\hline$F_{1}$ & $C_{33}=0, M_{11}=M_{12}$ & $e^{i\left(\phi_{e}-\phi_{\mu}\right)}$ & $M_{33}=0, C_{11}=C_{12}$ & $e^{i\left(\phi_{\mu}-\phi_{e}\right)}$ \\
\hline$F_{2}$ & $C_{33}=0, M_{11}=M_{13}$ & $e^{i\left(\phi_{e}-\phi_{\tau}\right)}$ & $M_{33}=0, C_{11}=C_{13}$ & $e^{i\left(\phi_{\tau}-\phi_{e}\right)}$ \\
\hline$F_{3}$ & $C_{33}=0, M_{11}=M_{22}$ & $e^{2 i\left(\phi_{e}-\phi_{\mu}\right)}$ & $M_{33}=0, C_{11}=C_{22}$ & $e^{2 i\left(\phi_{\mu}-\phi_{e}\right)}$ \\
\hline$F_{4}$ & $C_{33}=0, M_{11}=M_{23}$ & $e^{i\left(2 \phi_{e}-\phi_{\mu}-\phi_{\tau}\right)}$ & $M_{33}=0, C_{11}=C_{23}$ & $e^{i\left(-2 \phi_{e}+\phi_{\mu}+\phi_{\tau}\right)}$ \\
\hline$F_{5}$ & $C_{33}=0, M_{12}=M_{13}$ & $e^{i\left(\phi_{\mu}-\phi_{\tau}\right)}$ & $M_{33}=0, C_{12}=C_{13}$ & $e^{i\left(\phi_{\tau}-\phi_{\mu}\right)}$ \\
\hline$F_{6}$ & $C_{33}=0, M_{12}=M_{22}$ & $e^{i\left(\phi_{e}-\phi_{\mu}\right)}$ & $M_{33}=0, C_{12}=C_{22}$ & $e^{i\left(\phi_{\mu}-\phi_{e}\right)}$ \\
\hline$F_{7}$ & $C_{33}=0, M_{12}=M_{23}$ & $e^{i\left(\phi_{e}-\phi_{\tau}\right)}$ & $M_{33}=0, C_{12}=C_{23}$ & $e^{i\left(\phi_{\tau}-\phi_{e}\right)}$ \\
\hline$F_{8}$ & $C_{33}=0, M_{13}=M_{22}$ & $e^{i\left(\phi_{e}+\phi_{\tau}-2 \phi_{\mu}\right)}$ & $M_{33}=0, C_{13}=C_{22}$ & $e^{i\left(-\phi_{e}-\phi_{\tau}+2 \phi_{\mu}\right)}$ \\
\hline$F_{9}$ & $C_{33}=0, M_{13}=M_{23}$ & $e^{i\left(\phi_{e}-\phi_{\mu}\right)}$ & $M_{33}=0, C_{13}=C_{23}$ & $e^{i\left(\phi_{\mu}-\phi_{e}\right)}$ \\
\hline$F_{10}$ & $C_{33}=0, M_{22}=M_{23}$ & $e^{i\left(\phi_{\mu}-\phi_{\tau}\right)}$ & $M_{33}=0, C_{22}=C_{23}$ & $e^{i\left(\phi_{\tau}-\phi_{\mu}\right)}$ \\
\hline
\end{tabular}

On the other hand, only four $A_{1}, A_{4}, A_{5}$, and $A_{6}$ seem to be viable with current oscillation data for type $\mathrm{Y}$, while inverted mass spectrum (IS) is ruled out for these cases.

For both types $\mathrm{X}$ and $\mathrm{Y}$, no noticeable constraint has been found on the parameter space of CP-violating phases $(\rho, \sigma, \delta)$. For type $\mathrm{X}$, all the viable cases predict the value of $|M|_{e e}$ in the range of $0.01 \mathrm{eV}$ to $0.05 \mathrm{eV}$. This prediction lies well within the sensitivity limit of neutrinoless double-beta decay experiments [49-51]. On the other hand, for type $\mathrm{Y},|M|_{e e}$ is predicted to be zero implying that neutrinoless doublebeta decay is forbidden. Also the lower bound on lowest neutrino mass $\left(m_{o}\right)$ is found to be extremely small $\left(\sim 10^{-3}\right.$ or less) for all the viable cases of type $\mathrm{X}$ and type $\mathrm{Y}$ structure (Table 3). For the purpose of illustration, we have presented the correlation plots for $A_{1}$ indicating the parameter space of $\rho, \sigma, \delta,|M|_{e e}$, and lowest neutrino mass $\left(m_{o}\right)$ (Figures 1 and 2).

Category B (C). In Category B, all the ten possible cases are allowed for both type $\mathrm{X}$ and type $\mathrm{Y}$ structure, respectively, at $3 \sigma$ CL (Table 4 ). Cases $B_{2,3,4,5,8,9,10}$ allow both NS and IS for type X, while cases $B_{1,2,3,4,5,8,9,10}$ allow both NS and IS for type $\mathrm{Y}$. As mentioned earlier, cases of Category B are related to cases belonging to Category $\mathrm{C}$ via permutation symmetry; therefore we can obtain the results for Category $\mathrm{C}$ from B by using (41).

Type X cases $B_{1}$ (IS), $B_{2}$ (IS), $B_{3}$ (NS, IS), $B_{4}$ (IS), $B_{5}$ (NS, IS), $B_{7}$ (NS), $B_{8}$ (IS), $B_{9}$ (NS, IS), $B_{10}$ (IS), $C_{1}$ (IS), $C_{2}$ (IS), $\mathrm{C}_{4}$ (IS), $C_{5}$ (NS, IS), $C_{6}$ (NS, IS), $C_{7}$ (NS), $C_{8}$ (IS), $C_{8}$ (IS), $C_{9}$ (NS, IS), and $C_{10}$ (IS) cover literally the complete range of $\delta$. However, for $B_{2}$ (NS), $B_{4}$ (NS), $B_{6}(\mathrm{NS}), B_{8}(\mathrm{NS}), B_{10}(\mathrm{NS})$, $C_{3}(\mathrm{NS}), C_{5}(\mathrm{NS}), C_{7}(\mathrm{NS}), C_{8}(\mathrm{NS})$, and $C_{10}$ (NS) the parameter space of $\delta$ is found to be reduced to an appreciable extent (Table 4).

On the other hand, type Y cases $B_{1}$ (NS), $B_{2}$ (NS), $B_{3}$ (NS, IS), $B_{4}$ (NS), $B_{5}$ (NS, IS), $B_{7}$ (IS), $B_{8}$ (NS), $B_{9}$ (NS, IS), $B_{10}$ (NS), $C_{1}$ (NS), $C_{2}$ (NS), $C_{4}$ (NS), $C_{5}$ (NS, IS), $C_{6}$ (NS, IS), $C_{7}$ (IS), $C_{8}$ (NS), $C_{8}$ (NS), $C_{9}$ (NS, IS), and $C_{10}$ (NS) cover approximately the complete range of $\delta$. For $B_{1}$ (IS), $B_{2}$ (IS), $B_{4}$ (IS), $B_{6}$ (IS), $B_{8}$ (IS), $B_{10}$ (IS), $C_{1}$ (IS), $C_{3}$ (IS), $C_{5}$ (IS), $C_{7}$ (IS), $C_{8}$ (IS), and $C_{10}$ (IS), the parameter space of $\delta$ is found to be constricted (Table 4).

From the analysis, it is found that textures $B_{2}, B_{4}, C_{5}$, and $C_{7}$ belonging to type $\mathrm{X}$ predict near maximal Dirac type $\mathrm{CP}$ violation (i.e., $\delta \approx 90^{\circ}$ and $270^{\circ}$ ) for NS. In addition, the Majorana phases $\rho$ and $\sigma$ are found to be very close to $0^{0}$ for these cases. On the other hand, in case of type $\mathrm{Y}, B_{1}, B_{2}, B_{4}, B_{6}, C_{1}, C_{4}, C_{5}$ and $C_{7}$ show almost similar constraints on the parameter space for $\delta$ however for opposite mass spectrum (Table 4). In Figures 3, 4, 5, and 6, we have complied the correlation plots for case $B_{2}$ for both types X and $Y$ comprising the unknown parameters $\rho, \sigma, \delta,|M|_{e e}$, and lowest neutrino mass $\left(m_{o}\right)$. As explicitly shown in Figures 3(a), 3(b), and 6(b), $\delta \approx 90^{\circ}$ and $270^{\circ}$, while $\rho, \sigma \approx 0^{\circ}$. The correlation plots between $|M|_{e e}$ and $m_{o}$ have been encapsulated in Figures 3(c), 4(c), 5(c), and 6(c). The plots indicate the strong linear relation correlation between these parameters and, in addition, the lower bound on both the parameters is somewhere in the range from 0.001 to $0.01 \mathrm{eV}$. The prediction for the allowed space of $|M|_{e e}$ for all the cases of category B is given in Table 4.

Category $D(F)$. In Category $\mathrm{D}$, only nine cases are acceptable with neutrino oscillation data at $3 \sigma$ CL for both type X and type $\mathrm{Y}$ structures, respectively, while case $D_{8}$ is excluded for both of them (Table 5). Cases $D_{1}, D_{2}, D_{4}, D_{5}, D_{6}, D_{7}$, and $D_{9}$ show both NS and IS for type X and type Y, respectively, while $D_{3}$ and $D_{10}$ are acceptable for IS (NS) and NS(IS), respectively, in case of type $\mathrm{X}$ (type $\mathrm{Y}$ ) structure. Similarly, the results for cases belonging to Category $\mathrm{F}$ can be obtained from Category $\mathrm{D}$ since both are related via permutation symmetry. It is found that only nine cases are allowed with data in category $\mathrm{F}$, while $F_{7}$ is excluded at $3 \sigma \mathrm{CL}$.

Cases $D_{1}(\mathrm{NS}), D_{2}$ (NS, IS), $D_{3}$ (IS), $D_{4}(\mathrm{NS}), D_{5}(\mathrm{NS}$, IS), $D_{6}(\mathrm{NS}), D_{7}(\mathrm{NS}), D_{9}(\mathrm{NS}), D_{10}(\mathrm{NS}), F_{1}(\mathrm{NS}), F_{2}$ (NS, IS), $F_{3}$ (IS), $F_{4}(\mathrm{NS}), F_{5}(\mathrm{NS}, \mathrm{IS}), F_{6}(\mathrm{NS}), F_{7}(\mathrm{NS}), F_{9}(\mathrm{NS})$, and 
TABLE 3: The allowed ranges of Dirac CP-violating phase $\delta$, the Majorana phases $\rho, \sigma$, effective neutrino mass $|M|_{e e}$, and lowest neutrino mass $m_{0}$ for the experimentally allowed cases of Category A at $3 \sigma \mathrm{CL}$. The predictions corresponding to $\left(\lambda_{13}\right)_{-}$and $\left(\lambda_{23}\right)_{-}$neutrino mass ratios have been put into brackets.

\begin{tabular}{|c|c|c|c|c|}
\hline \multirow[b]{2}{*}{ Cases } & \multicolumn{2}{|r|}{$\mathrm{X}$} & \multicolumn{2}{|l|}{$\mathrm{Y}$} \\
\hline & NS & IS & NS & IS \\
\hline \multirow[t]{8}{*}{$A_{1}$} & $\times$ & $\rho=-90^{0}--72^{0} \oplus 72^{0}-90^{0}$ & $\rho=-90^{0}-90^{0}$ & $x$ \\
\hline & $x$ & $=\left(-90^{0}--72^{0} \oplus 72^{0}-90^{0}\right)$ & $=\left(-90^{0}-90^{0}\right)$ & $x$ \\
\hline & $x$ & $\sigma=-90^{\circ}-90^{\circ}$ & $\sigma=-90^{0}-90^{0}$ & $x$ \\
\hline & $x$ & $=\left(-90^{0}-90^{\circ}\right)$ & $=\left(-90^{0}-90^{0}\right)$ & $x$ \\
\hline & $x$ & $\delta=0^{0}-93.2^{0} \oplus 116.6^{0}-247.7^{0} \oplus 268.8^{0}-360^{0}$ & $\delta=0^{0}-360^{0}$ & $x$ \\
\hline & $x$ & $=\left(0^{0}-166.27^{0} \oplus 191.98^{0}-360^{0}\right)$ & $=\left(0^{0}-360^{0}\right)$ & $x$ \\
\hline & $x$ & $|M|_{e e}=0.0114-0.0540$ & $|M|_{e e}=0.0$ & $x$ \\
\hline & $x$ & $m_{0}=0.000820-0.0470$ & $m_{0}=0.00155-0.0103$ & $x$ \\
\hline \multirow[t]{8}{*}{$A_{2}\left(A_{8}\right)$} & $x$ & $\rho=-85.6^{0}--76.3^{0} \oplus 76.4^{0}-85.6^{0}$ & $\times$ & $x$ \\
\hline & $x$ & $=\left(-85.4^{0}--78.1^{0} \oplus 78.2^{0}-85.1^{0}\right)$ & $x$ & $x$ \\
\hline & $x$ & $\sigma=-70.1^{0}--43.1^{0} \oplus 43.1^{0}-70^{0}$ & $x$ & $x$ \\
\hline & $x$ & $=\left(-70.1^{0}--44.2^{0} \oplus 43.3^{0}-76^{0}\right)$ & $x$ & $x$ \\
\hline & $x$ & $\delta=0^{0}-360^{0}$ & $x$ & $x$ \\
\hline & $x$ & $=\left(0^{0}-360^{0}\right)$ & $\times$ & $x$ \\
\hline & $x$ & $|M|_{e e}=0.0273-0.0489$ & $x$ & $x$ \\
\hline & $x$ & $m_{0}=0.000820-0.0484$ & $\times$ & $x$ \\
\hline \multirow[t]{8}{*}{$A_{3}\left(A_{7}\right)$} & $\times$ & $\rho=-81.94^{0}--75.9^{0} \oplus 75.4^{0}-81.93^{0}$ & $\times$ & $x$ \\
\hline & $x$ & $=\left(-82.4^{0}--77.1^{0} \oplus 76.9^{0}-82^{0}\right)$ & $x$ & $x$ \\
\hline & $x$ & $\sigma=-70.1^{0}--52.1^{0} \oplus 50.47^{0}-66.41^{0}$ & $x$ & $x$ \\
\hline & $x$ & $=\left(-72^{0}--54.2^{0} \oplus 53.3^{0}-73^{0}\right)$ & $x$ & $x$ \\
\hline & $x$ & $\delta=0^{0}-360^{\circ}$ & $x$ & $x$ \\
\hline & $x$ & $=\left(0^{0}-360^{0}\right)$ & $x$ & $x$ \\
\hline & $x$ & $|M|_{e e}=0.0308-0.0451$ & $x$ & $x$ \\
\hline & $x$ & $m_{0}=0.000969-0.00187$ & $\times$ & $x$ \\
\hline \multirow[t]{8}{*}{$A_{4}\left(A_{6}\right)$} & $x$ & $\rho=-89.8^{0}--71.2^{0} \oplus 71.1^{0}-89.9^{0}$ & $\rho=-90^{0}-90^{0}$ & $x$ \\
\hline & $\times$ & $=\left(-90^{0}--72.1^{0} \oplus 72^{0}-90^{0}\right)$ & $=\left(-90^{0}-90^{0}\right)$ & $x$ \\
\hline & $x$ & $\sigma=-90^{\circ}-90^{\circ}$ & $\sigma=-90^{\circ}-90^{\circ}$ & $x$ \\
\hline & $x$ & $=\left(-90^{0}-90^{\circ}\right)$ & $=\left(-90^{0}-90^{0}\right)$ & $x$ \\
\hline & $x$ & $\delta=29.78^{0}-89.64^{0} \oplus 148.6^{0}-208^{0} \oplus 269.8^{0}-329^{0}$ & $\delta=0^{0}-360^{0}$ & $x$ \\
\hline & $\times$ & $=\left(0^{0}-29.78^{0} \oplus 90.98^{0}-151.64^{0} \oplus 208.6^{0}-271^{0} \oplus 329.8^{0}-360^{0}\right)$ & $\delta=0^{0}-360^{0}$ & $x$ \\
\hline & $\times$ & $|M|_{e e}=0.0108-0.0501$ & $|M|_{e e}=0.0$ & $x$ \\
\hline & $\times$ & $m_{0}=0.000904-0.00440$ & $m_{0}=0.00143-0.0103$ & $x$ \\
\hline \multirow[t]{8}{*}{$A_{5}\left(A_{5}\right)$} & $x$ & $\rho=-90^{0}--71^{0} \oplus 71^{0}-90^{0}$ & $\rho=-90^{0}--42.3^{0} \oplus 41.5^{0}-90^{0}$ & $x$ \\
\hline & $x$ & $=\left(-90^{0}--71.2^{0} \oplus 72^{0}-90^{0}\right)$ & $=\left(-56.6^{0}-56.7^{0}\right)$ & $x$ \\
\hline & $x$ & $\sigma=-90^{\circ}-90^{0}$ & $\sigma=-62.5^{0}-62.4^{0}$ & $x$ \\
\hline & $x$ & $=\left(-90^{\circ}-90^{\circ}\right)$ & $=\left(-90^{0}--41.2^{0} \oplus 41.4^{0}-90^{0}\right)$ & $x$ \\
\hline & $x$ & $\delta=0^{0}-44^{0} \oplus 92^{0}-162^{0} \oplus 198^{0}-268^{0} \oplus 327^{0}-360^{0}$ & $\delta=0^{0}-360^{\circ}$ & $x$ \\
\hline & $x$ & $=\left(27.36^{0}-109.3^{0} \oplus 147.9^{0}-209.37^{0} \oplus 250.7^{0}-331^{0}\right)$ & $=0^{0}-360^{0}$ & $x$ \\
\hline & $\times$ & $|M|_{e e}=0.0113-0.0500$ & $|M|_{e e}=0.0$ & $x$ \\
\hline & $x$ & $m_{0}=0.000822-0.00440$ & $m_{0}=0.00306-0.0105$ & $x$ \\
\hline \multirow[t]{8}{*}{$A_{9}\left(A_{10}\right)$} & $x$ & $\rho=-84.2^{0}--74.53^{0} \oplus 73.52^{0}-84.2^{0}$ & $\times$ & $x$ \\
\hline & $x$ & $=\left(-84.2^{0}--75^{0} \oplus 75^{0}-85.1^{0}\right)$ & $x$ & $x$ \\
\hline & $x$ & $\sigma=-74.83^{0}--50.3^{0} \oplus 50.1^{0}-73.4^{0}$ & $x$ & $x$ \\
\hline & $x$ & $=\left(-75^{0}--50.3^{0} \oplus 50.1^{0}-75^{0}\right)$ & $x$ & $x$ \\
\hline & $\times$ & $\delta=0^{0}-360^{\circ}$ & $x$ & $x$ \\
\hline & $x$ & $=\left(0^{0}-360^{0}\right)$ & $x$ & $x$ \\
\hline & $x$ & $|M|_{e e}=0.0293-0.0469$ & $x$ & $x$ \\
\hline & $\times$ & $m_{0}=0.000940-0.00190$ & $\times$ & $\times$ \\
\hline
\end{tabular}




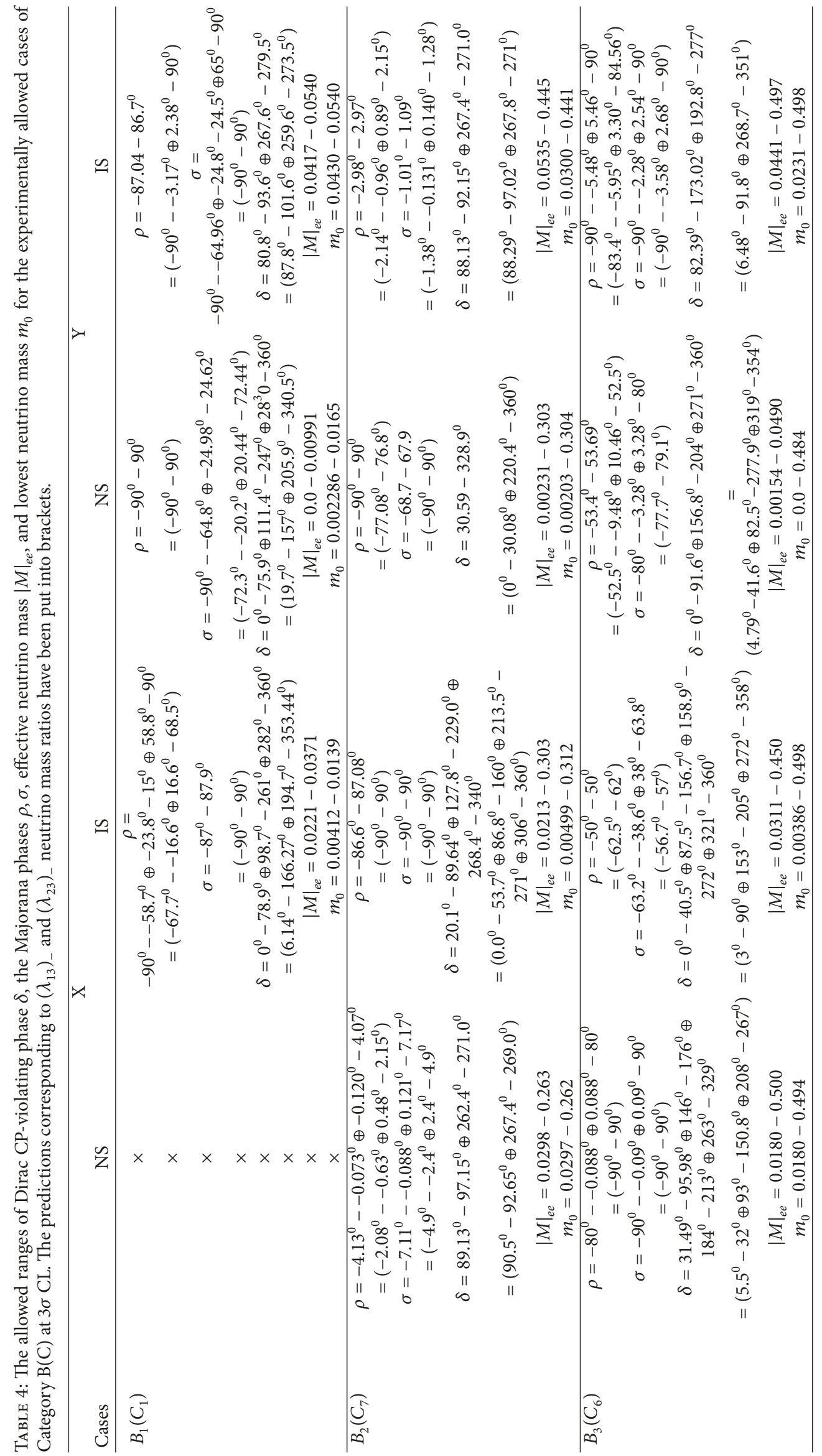




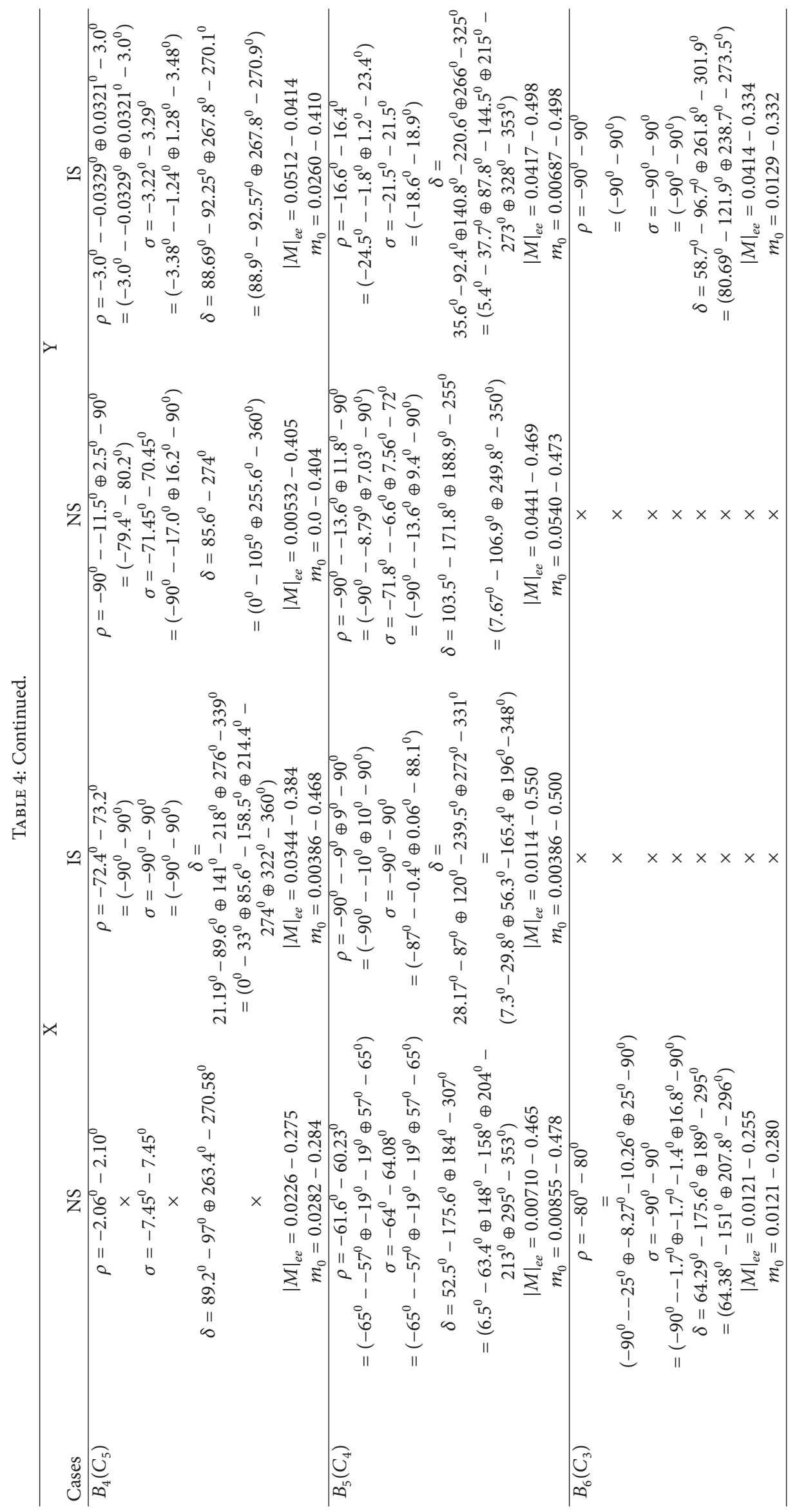




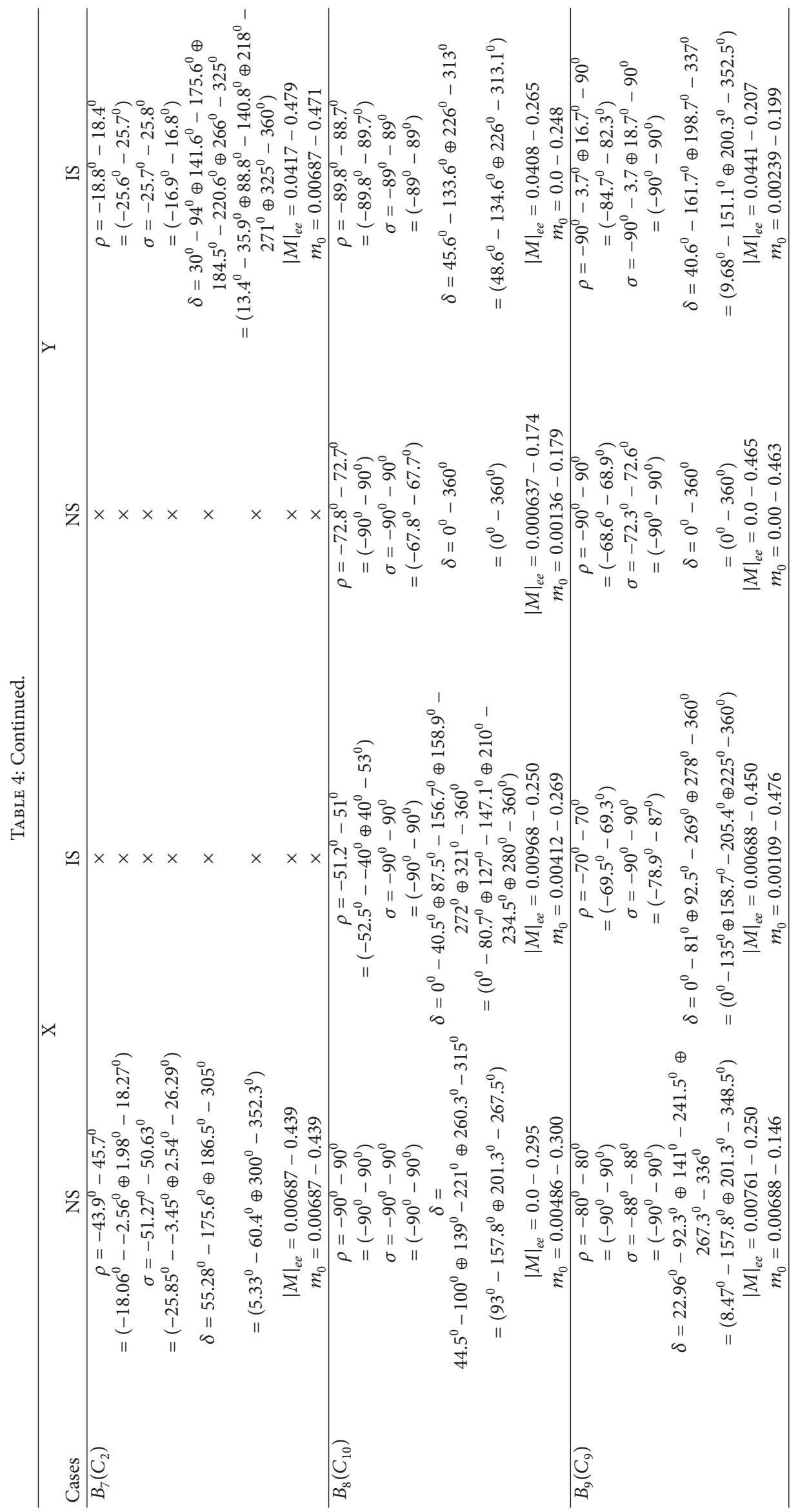




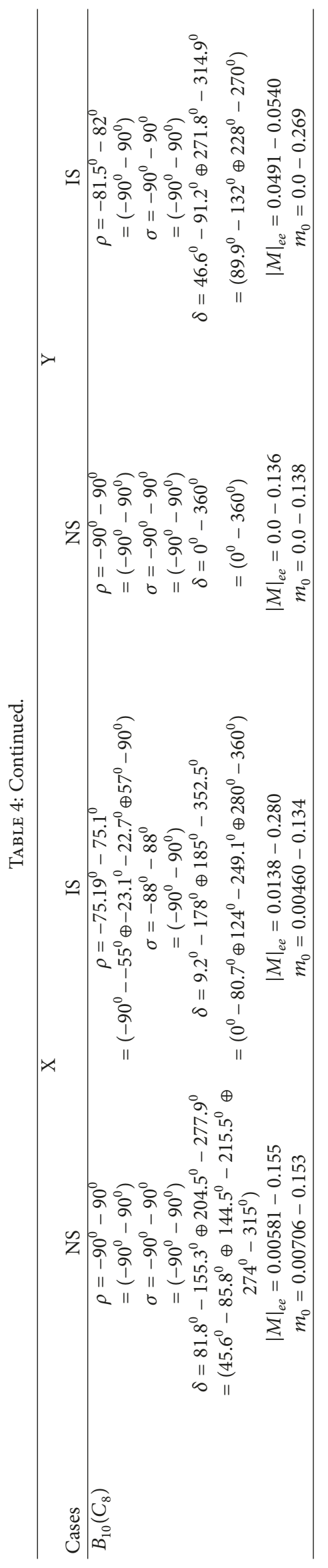




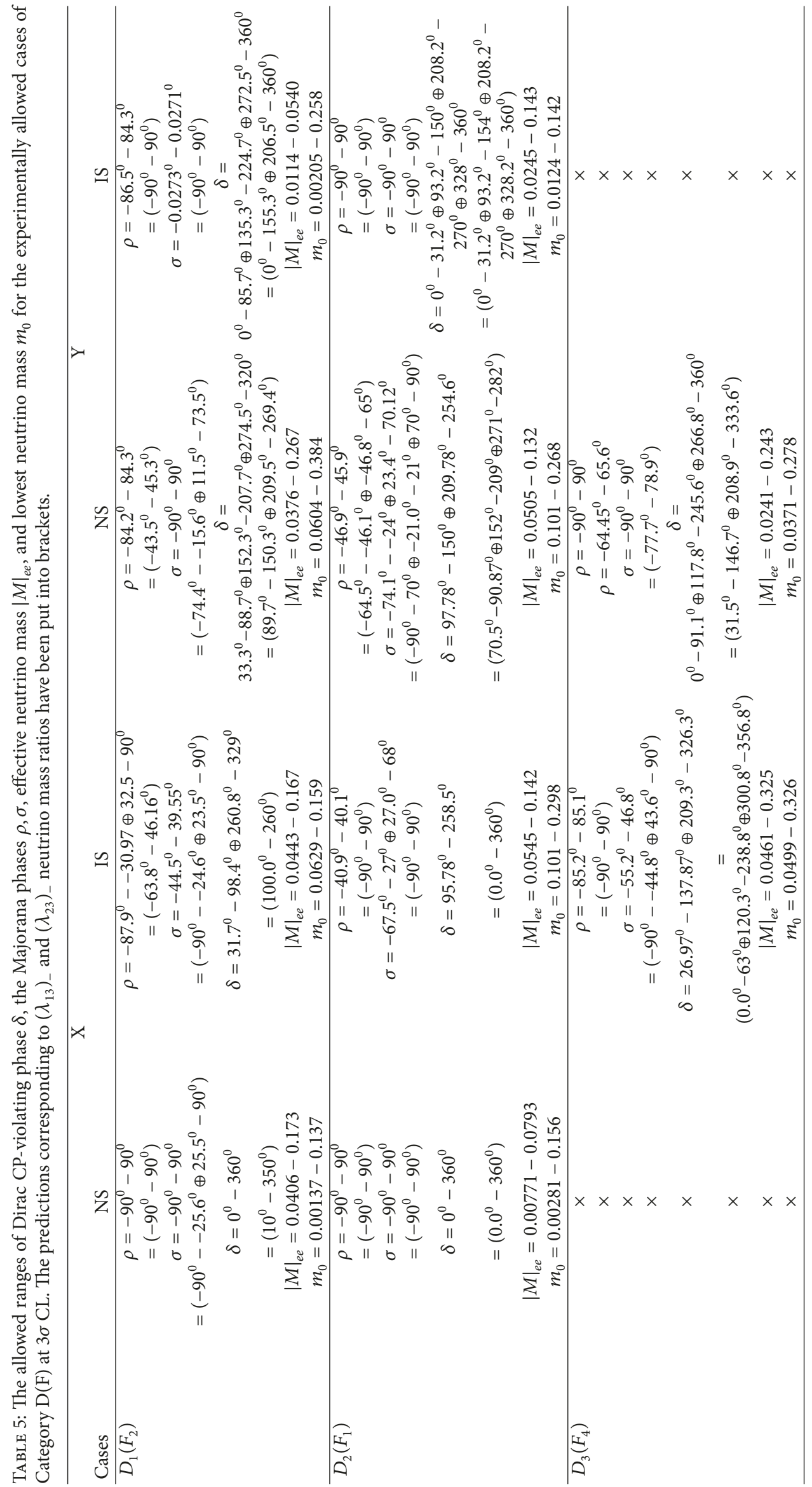




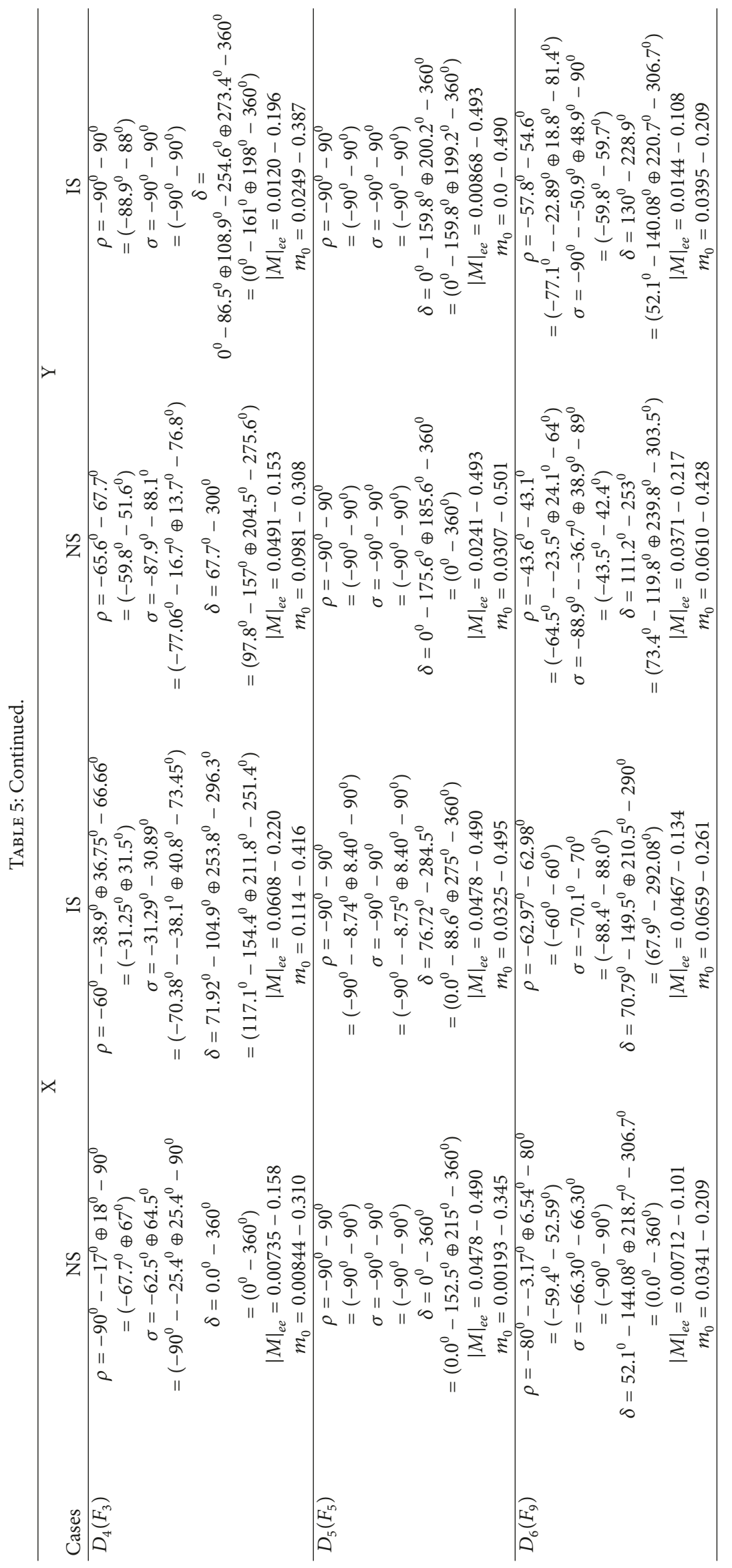




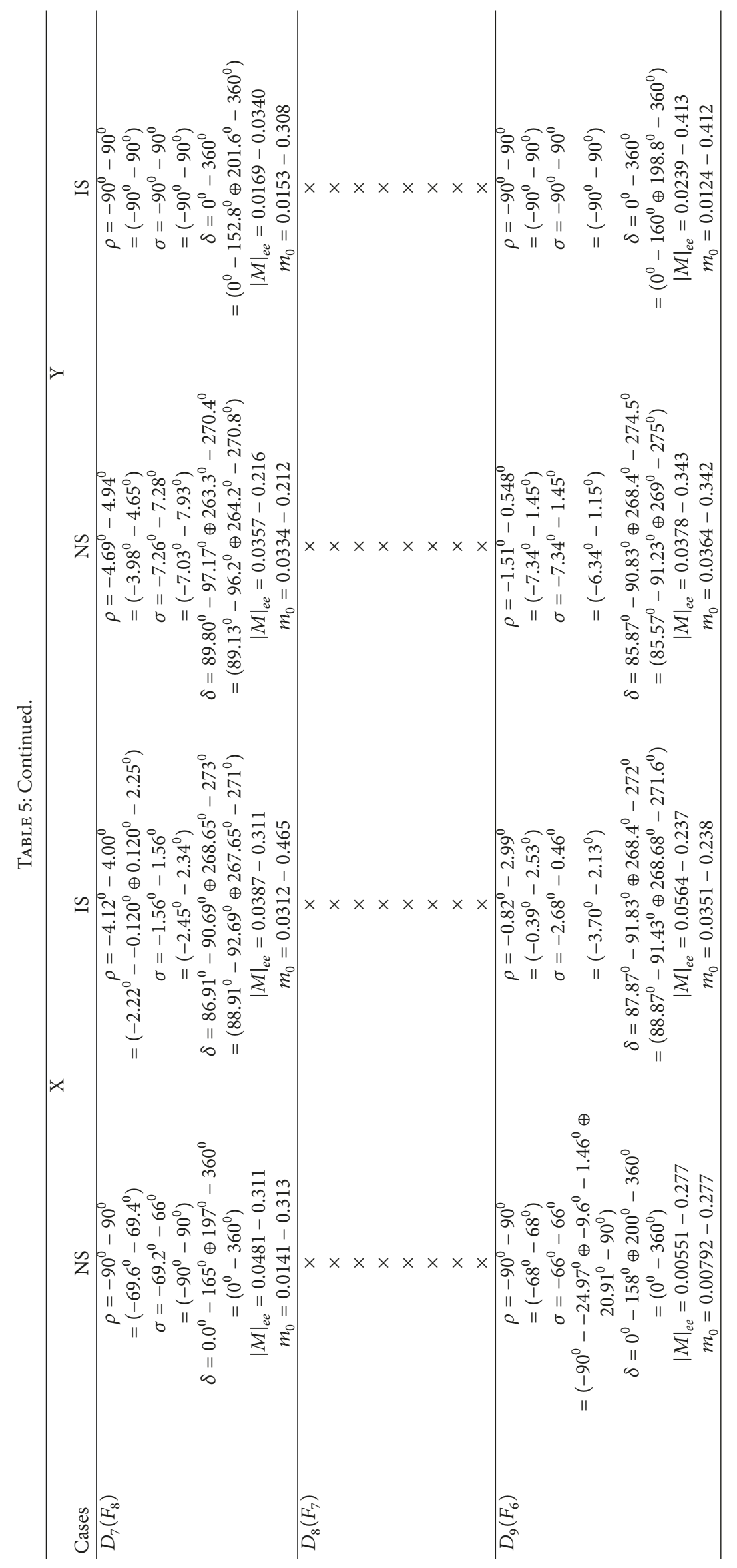




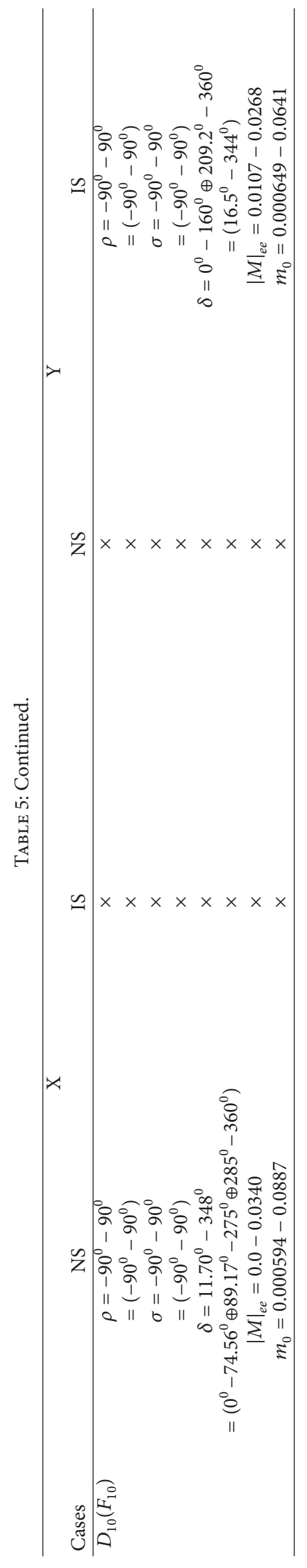




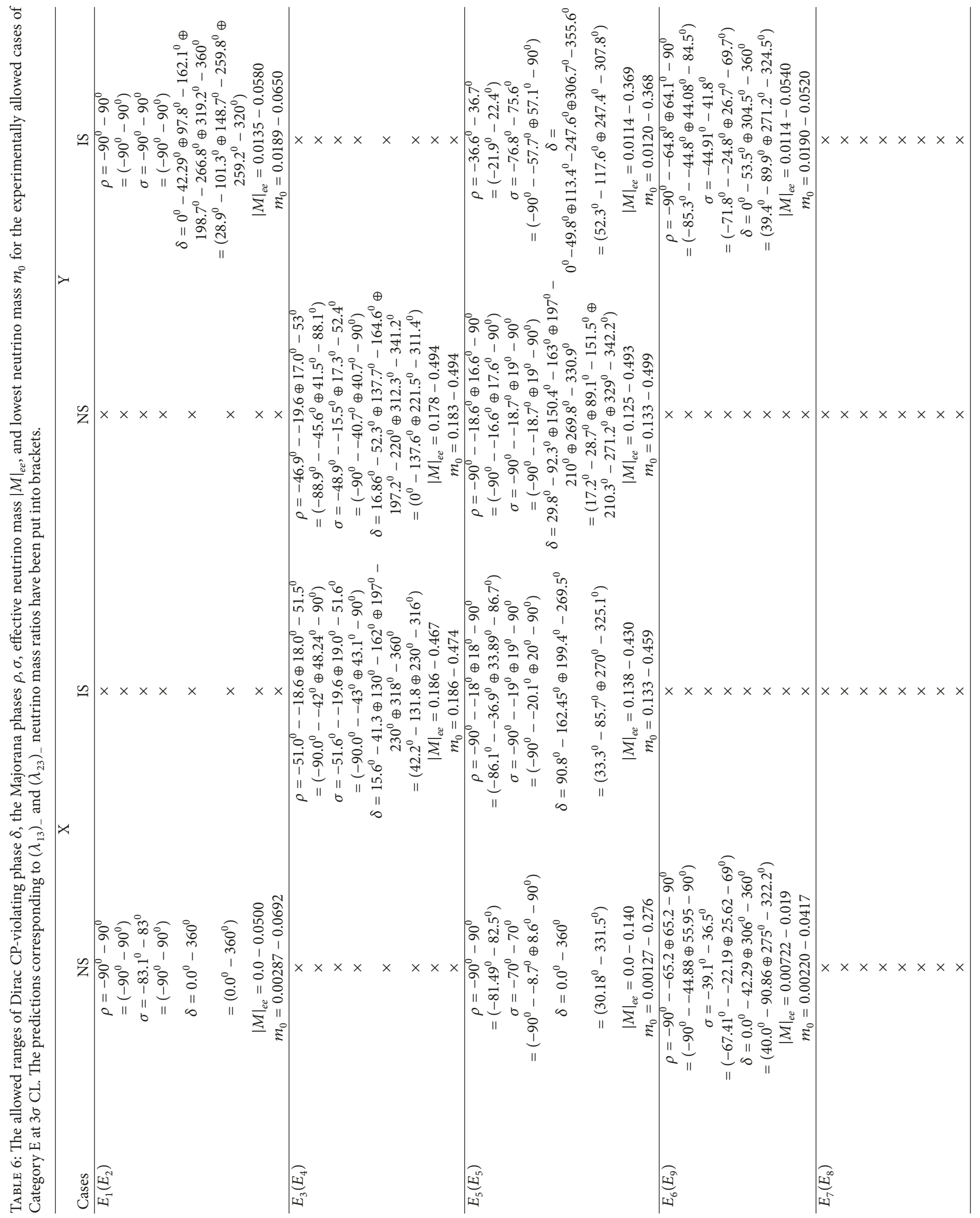




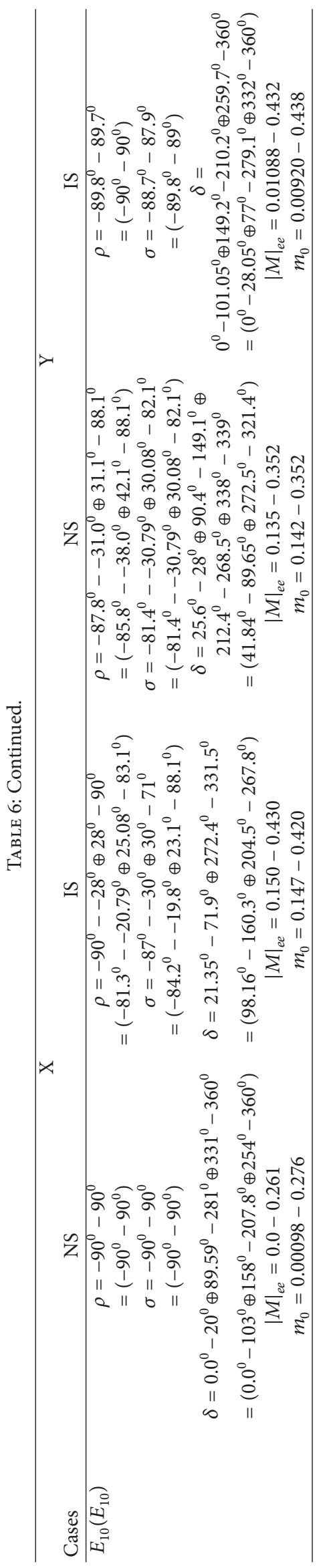


$F_{10}$ (NS) predict literally no constraints on $\delta$ for type X texture. These cases give identical predictions for type $\mathrm{Y}$ as well, however for opposite mass ordering. On the other hand, for cases $D_{6}$ (IS), $D_{4}$ (IS), $D_{7}$ (IS), $D_{9}$ (IS), $F_{3}$ (IS), $F_{6}$ (IS), $F_{8}$ (IS), and $F_{9}(\mathrm{IS}), \delta$ is notably constrained for type $\mathrm{X}$ and similar observations have been found for these cases in type Y, however for opposite mass ordering (Table 5).

It is found that textures $D_{7}$ (IS), $D_{9}$ (IS), $F_{6}$ (IS), and $F_{8}$ (IS) belonging to type $\mathrm{X}$ predict near maximal Dirac CP violation (i.e., $\delta \approx 90^{\circ}$ and $270^{\circ}$ ). In addition, the Majorana phases $\rho$ and $\sigma$ are found to be very close to $0^{0}$ for these cases. The similar predictions hold for these cases belonging to type $\mathrm{Y}$ structure however for opposite mass spectrum.

The prediction on the allowed range of $|M|_{e e}$ for all the cases of category D is provided in Table 5. As an illustration, in Figures 7, 8, 9, and 10 we have complied the correlation plots for case $D_{7}$ for type $\mathrm{X}$ and type $\mathrm{Y}$ structures. Figures 7(a), 7(b), 10(a), and 10(b) indicate no constraint on $\delta, \rho, \sigma$ for NS(IS) corresponding to type X (type Y) structure at $3 \sigma \mathrm{CL}$. On the other hand, $\delta \approx 90^{\circ}$ and $270^{\circ}$, while $\rho$ and $\sigma$ approach to $0^{0}$ for IS in case of type X structure (Figure 8). However, similar predictions for $\delta, \rho, \sigma$ have been observed for type Y, however for NS (Figure 9). In Figures 7(c), 8(c), 9(c), and 10 (c), we have presented the correlation plots between $|M|_{e e}$ and $m_{0}$ indicating the linear correlation.

Category E. In Category E, only eight out of ten cases are allowed with experimental data for both type $\mathrm{X}$ and type $\mathrm{Y}$ structures at $3 \sigma \mathrm{CL}$ (Table 6). Cases $E_{7}$ and $E_{8}$ are ruled out for both type $\mathrm{X}$ and type $\mathrm{Y}$ structures. Only $E_{5}$ and $E_{10}$ favor both NS and IS, while rest of the cases favor either NS or IS for type X and type $\mathrm{Y}$ structure (Table 6). From Table 6 , it is clear that $E_{1}, E_{2}, E_{3}, E_{4}, E_{5}, E_{10}$ cover literally full range of $\delta$ for type $\mathrm{X}$. Same cases show identical prediction for type $\mathrm{Y}$, however for opposite mass spectrum. For NS, cases $E_{1}, E_{2}, E_{5}, E_{9}, E_{10}$ belonging to type X predict the lower bound on effective mass $|M|_{e e}$ to be zero, while for IS, cases $E_{3}, E_{4}, E_{5}, E_{10}$ predict larger lower bound (greater than $0.01 \mathrm{eV}$ ) on $|M|_{e e}$ (Table 6). However for type $\mathrm{Y}$, all these cases show larger lower bound on $|M|_{e e}(\geq 0.01 \mathrm{eV})$ for both NS and IS.

For the purpose of illustration, we have presented the correlation plots for $E_{1}$ indicating the parameter space of $\rho, \sigma, \delta,|M|_{e e}$, and lowest neutrino mass $\left(m_{o}\right)$ (Figures 11 and 12). As shown in Figures 11 and $12, \rho, \sigma, \delta$ remain literally unconstrained for both type $\mathrm{X}$ and type $\mathrm{Y}$ structures. In addition, there is a linear correlation among $\rho, \sigma$, and $\delta$ at $3 \sigma$ CL for type $\mathrm{X}$ structure (Table 6 ). Figure $11(\mathrm{c})$ indicates the strong linear correlation between $|M|_{e e}$ and $m_{o}$ and, in addition, the lower bound on $|M|_{e e}$ is predicted to be zero.

\section{Summary and Conclusion}

To summarize, we have discussed the novel possibilities of hybrid textures in the flavor basis wherein the assumption of either one zero minor and an equality between the elements or one zero element and an equality between the cofactors in the Majorana neutrino mass matrix is considered. Out of sixty phenomenologically possible cases, only 56 are found to be viable for type $\mathrm{X}$, while only 50 are viable with the present data for type $\mathrm{Y}$ at $3 \sigma \mathrm{CL}$. Therefore, out of 120 only 106 cases are found to be viable with the existing data. However only 38 seems to restrict the parametric space of CP-violating phases $\delta, \rho$, and $\sigma$, while 16 out of these predict near maximal Dirac $\mathrm{CP}$ violation, i.e., $\delta \simeq 90^{\circ}, 270^{\circ}$. The allowed parameter space for effective mass term $|M|_{e e}$ related to neutrinoless doublebeta decay and lowest neutrino mass term for all viable cases have been carefully studied. The present viable cases may be derived from the discrete symmetry. However the symmetry realization for each case in a systematic and self-consistent way deserves fine-grained research. The viability of these cases suggests that there are still rich unexplored structures of the neutrino mass matrix from both the phenomenological and the theoretical points of view.

To conclude our discussion, we would like to add that the hybrid textures comprising either one zero element and an equality between the elements or one zero minor and an equality between the cofactors lead to 106 viable cases; therefore there are now total 212 viable cases pertaining to the hybrid textures of $M_{\nu}$ in the flavor basis. Since most of these cases overlap in their predictions regarding the experimentally undetermined parameters, therefore we expect that only the future long baseline experiments, neutrinoless doublebeta decay experiments, and cosmological observations could help us select the appropriate structure of mass texture.

\section{Data Availability}

The data used to support the findings of this study are available from the corresponding author upon request.

\section{Conflicts of Interest}

The authors declare that there are no conflicts of interest regarding the publication of this paper.

\section{Acknowledgments}

The author would like to thank the Director of the National Institute of Technology Kurukshetra, for providing the necessary facilities to work.

\section{References}

[1] Abe. K., N. Abgrall, Y. Ajima et al., "Indication of electron neutrino appearance from an accelerator-produced off-axis muon neutrino beam," Physical Review Letters, vol. 107, Article ID 041801, 2011.

[2] P. Adamson, D. J. Auty, D. S. Ayres et al., "Improved search for muon-neutrino to electron-neutrino oscillations in MINOS," Physical Review Letters, vol. 107, Article ID 181802, 2011.

[3] Y. Abe, C. Aberle, T. Akiri et al., "Indication for the disappearance of reactor electron antineutrinos in the Double Chooz experiment," Physical Review Letters, vol. 108, Article ID 131801, 2012. 
[4] F. P. An, J. Z. Bai, A. B. Balantekin et al., "Observation of electron-antineutrino disappearance at Daya Bay," Physical Review Letters, vol. 108, Article ID 171803, 2012.

[5] J. K. Ahn, S. Chebotaryov, J. H. Choi et al., "Observation of reactor electron antineutrinos disappearance in the RENO experiment," Physical Review Letters, vol. 108, Article ID 191802, 2012.

[6] S. Dev, R. R. Gautam, and L. Singh, "Neutrino mass matrices with two equalities between the elements or cofactors," Physical Review D, vol. 87, Article ID 073011, 2013.

[7] S. Dev, S. Verma, and S. Gupta, "Phenomenological analysis of hybrid textures of neutrinos," Physics Letters B, vol. 687, p. 53, 2010.

[8] J. Y. Liu and S. Zhou, "Hybrid textures of majorana neutrino mass matrix and current experimental tests," Physical Review, vol. 87, Article ID 093010, 2013.

[9] S. Kaneko, H. Sawanaka, and M. Tanimoto, "Hybrid textures of neutrinos," Journal of High Energy Physics, vol. 2005, no. 8, article 73, 2005.

[10] P. H. Frampton, S. L. Glashow, and D. Marfatia, "Zeroes of the neutrino mass matrix," Physics Letters B, vol. 536, no. 1-2, pp. 79-82, 2002.

[11] Z.-Z. Xing, "Texture zeros and majorana phases of the neutrino mass matrix," Physics Letters B, vol. 530, no. 1-4, pp. 159-166, 2002.

[12] B. R. Desai, D. P. Roy, and A. R. Vaucher, "Three-neutrino mass matrices with two texture zeros," Modern Physics Letters A, vol. 18, no. 20, pp. 1355-1365, 2003.

[13] S. Dev, S. Kumar, S. Verma, and S. Gupta, Nuclear Physics, vol. 784, p. 103, 2007.

[14] G. Ahuja, S. Kumar, M. Randhawa, M. Gupta, and S. Dev, "Texture 4 zero Fritzsch-like lepton mass matrices," Physical Review D: Particles, Fields, Gravitation and Cosmology, vol. 76, no. 1, Article ID 013006, 2007.

[15] S. Kumar, "Implications of a class of neutrino mass matrices with texture zeros for nonzero $\theta_{13}$," Physical Review D: Particles, Fields, Gravitation and Cosmology, vol. 84, no. 7, Article ID 077301, 2011.

[16] G. Blankenburg and D. Meloni, "Fine-tuning and naturalness issues in the two-zero neutrino mass textures," Nuclear Physics, vol. 867, p. 749, 2013.

[17] W. Grimus and P. O. Ludl, "Two-parameter neutrino mass matrices with two texture zeros," Journal of Physics G, vol. 40, Article ID 055003, 2013.

[18] M. Gupta and G. Ahuja, "Flavor mixings and textures of the fermion mass matrices," International Journal of Modern Physics, vol. 27, Article ID 1230033, 2012.

[19] J. Liao, D. Marfatia, and K. Whisnant, "Texture and Cofactor Zeros of the Neutrino Mass Matrix," Journal of High Energy Physics, vol. 13, 2014.

[20] D. Meloni, A. Meroni, and E. Peinado, "Two-zero Majorana textures in the light of the Planck results," Physical Review D: Particles, Fields, Gravitation and Cosmology, vol. 89, no. 5, Article ID 053009, 2014.

[21] P. O. Ludl and W. Grimus, "A complete survey of texture zeros in the lepton mass matrices," Journal of High Energy Physics, vol. 090, 2014.

[22] P. O. Ludl and W. Grimus, "A complete survey of texture zeros in general and symmetric quark mass matrices," Physics Letters $B$, vol. 744, pp. 38-42, 2015.
[23] M. Borah, D. Borah, and M. K. Das, "Discriminating majorana neutrino textures in the light of baryon asymmetry," Physical Review D, vol. 91, Article ID 113008, 2015.

[24] H. Fritzsch, Z. Z. Xing, and S. Zhou, "Two-zero textures of the Majorana neutrino mass matrix and current experimental tests," Journal of High Energy Physics, vol. 2011, article 83, 2011.

[25] R. Verma, "Exploring the predictability of symmetric texture zeros in quark mass matrices," Physical Review, vol. 96, Article ID 093010, 2017.

[26] S. Dev, L. Singh, and D. Raj, "Neutrino mass matrices with two vanishing elements/cofactors," The European Physical Journal C, vol. 75, no. 394, 2015.

[27] W. Wang, "Neutrino mass textures with one vanishing minor and two equal cofactors," The European Physical Journal C, vol. 73, article 2551, 2013.

[28] S. Dev, R. R. Gautam, and L. Singh, "Hybrid textures of the right-handed Majorana neutrino mass matrix," Physical Review D: Particles, Fields, Gravitation and Cosmology, vol. 88, Article ID 033008, 2013.

[29] L. Lavoura, "Zeros of the inverted neutrino mass matrix," Physics Letters B, vol. 609, no. 3-4, pp. 317-322, 2005.

[30] E. I. Lashin and N. Chamoun, "Zero minors of the neutrino mass matrix," Physical Review D: Particles, Fields, Gravitation and Cosmology, vol. 78, no. 7, Article ID 073002, 2008.

[31] E. I. Lashin, E. Malkawi, S. Nasri, and N. Chamoun, Physical Review D: Particles, Fields, Gravitation and Cosmology, vol. 80, no. 11, 2009.

[32] S. Dev, S. Gupta, R. R. Gautam, and L. Singh, "Near maximal atmospheric mixing in neutrino mass matrices with two vanishing minors," Physics Letters B, vol. 706, no. 2-3, pp. 168-176, 2011.

[33] T. Araki, J. Heeck, and J. Kubo, "Vanishing minors in the neutrino mass matrix from abelian gauge symmetries," Journal of High Energy Physics, vol. 2012, no. 7, 2012.

[34] J. Han, R. Wang, W. Wang, and X. Wei, "Neutrino mass matrices with one texture equality and one vanishing neutrino mass," Physical Review D: Particles, Fields, Gravitation and Cosmology, vol. 96, no. 7, 2017.

[35] S. Dev, D. Raj, and R. R. Gautam, "Neutrino mass matrices with three or four vanishing cofactors and nondiagonal charged lepton sector," Physical Review D: Particles, Fields, Gravitation and Cosmology, vol. 96, no. 9, 2017.

[36] W. Konetschny and W. Kummer, "Nonconservation of total lepton number with scalar bosons," Physics Letters B, vol. 70, no. 4, pp. 433-435, 1977.

[37] T. Cheng and L.-F. Li, "Neutrino masses, mixings, and oscillations in $\mathrm{SU}(2) \times \mathrm{U}(1)$ models of electroweak interactions," Physical Review D: Particles, Fields, Gravitation and Cosmology, vol. 22, no. 11, pp. 2860-2868, 1980.

[38] J. Schechter and J. W. F. Valle, "Neutrino masses in $\mathrm{SU}(2) \otimes \mathrm{U}(1)$ theories," Physical Review D: Particles, Fields, Gravitation and Cosmology, vol. 22, p. 2227, 1980.

[39] G. Lazarides, Q. Shafi, and C. Wetterich, Nuclear Physics, vol. 181, p. 287, 1981.

[40] R. N. Mohapatra and G. Senjanović, "Neutrino masses and mixings in gauge models with spontaneous parity violation," Physical Review D: Particles, Fields, Gravitation and Cosmology, vol. 23, article 165, 1981.

[41] P. Minkowski, " $\mu \longrightarrow \mathrm{e} \gamma$ at a rate of one out of 109 muon decays?" Physics Letters B, vol. 67, no. 4, pp. 421-428, 1977. 
[42] T. Yanagida, Proceedings of the Workshop on the Unified Theory and the Baryon Number in the Universe, O. Sawada and A. Sugamoto, Eds., KEK, Tsukuba, Japan, 1979.

[43] M. Gell-Mann, P. Ramond, and R. Slansky, "Complex spinors and unified theories in supergravity," P. Van Nieuwenhuizen and D. Z. Freedman, Eds., Amsterdam, Netherlands, 1979.

[44] R. N. Mohapatra and G. Senjanovic, "Neutrino mass and spontaneous parity nonconservation," Physical Review Letters, vol. 44, p. 912, 1980.

[45] W. Grimus, A. S. Joshipura, L. Lavoura, and M. Tanimoto, "Symmetry realization of texture zeros," The European Physical Journal C, vol. 36, no. 2, pp. 227-232, 2004.

[46] H. Fritzsch and Z. Xing, "How to describe neutrino mixing and CP violation,” Physics Letters B, vol. 517, no. 3-4, pp. 363-368, 2001.

[47] I. Esteban, M. C. Gonzalez-Garcia, M. Maltoni et al., "Updated fit to three neutrino mixing: exploring the accelerator-reactor complementarity," Journal of High Energy Physics, vol. 1, no. 87, 2017.

[48] P. F. de Salas, D. V. Forero, C. A. Ternes, M. Tortola, and J. W. F. Valle, "Status of neutrino oscillations 2018: first hint for normal mass ordering and improved CP sensitivity," Physics Letters B, vol. 782, pp. 633-640, 2018.

[49] J. Shirai, "Search for majorana neutrinos near the inverted mass hierarchy region with KamLAND-Zen," Physical Review Letters, vol. 117, Article ID 109903, 2016.

[50] J. B. Albert, D. J. Auty, and P. S. Barbeau, "Search for Majorana neutrinos with the first two years of EXO-200 data," Nature, vol. 510, pp. 229-234, 2014.

[51] S. Delloro, S. Marcocci, M. Viel, and F. Vissani, "Neutrinoless Double Beta Decay: 2015 Review," Advances in High Energy Physics, vol. 2016, Article ID 2162659, 37 pages, 2016.

[52] W. Rodejohann and J. Mod, "Neutrino-less Double Beta Decay and Particle Physics," International Journal of Modern Physics E, vol. 20, no. 9, 2011 . 

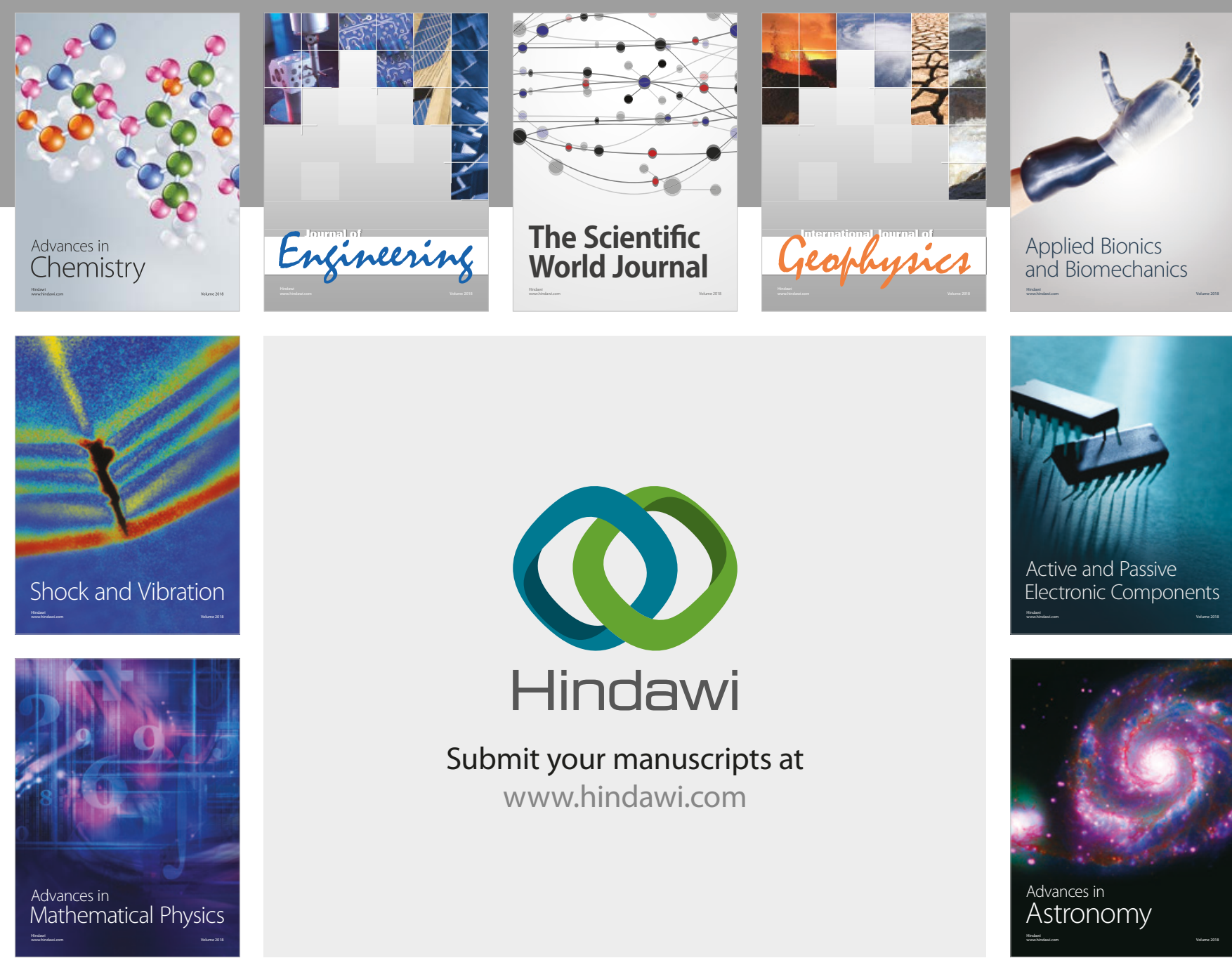

Submit your manuscripts at

www.hindawi.com

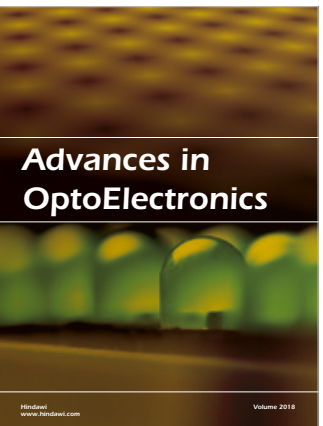

\section{Rotcting Machinery}
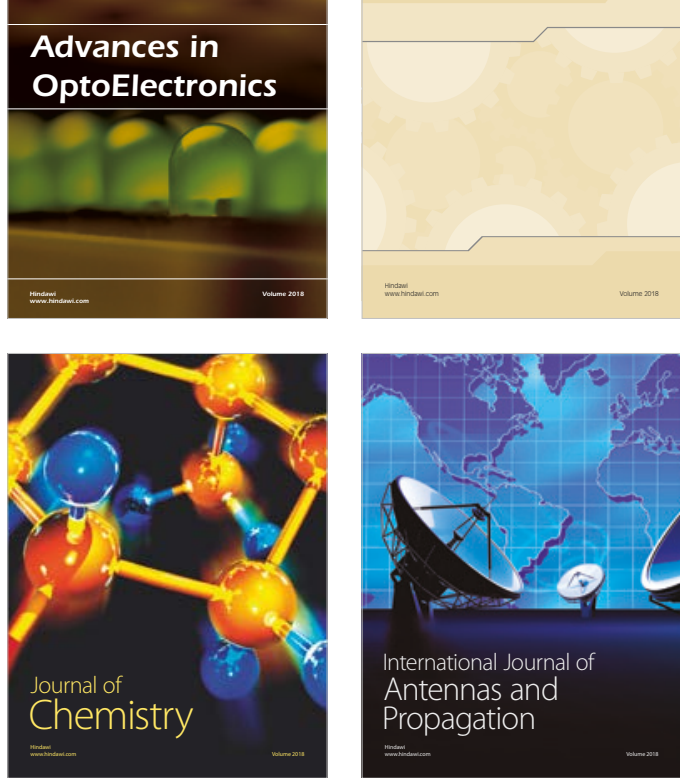

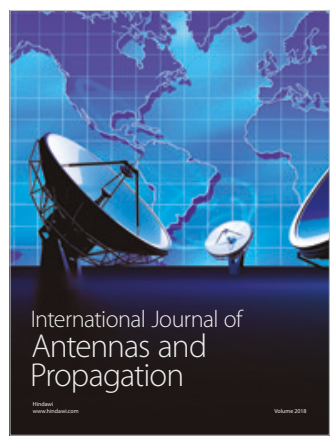

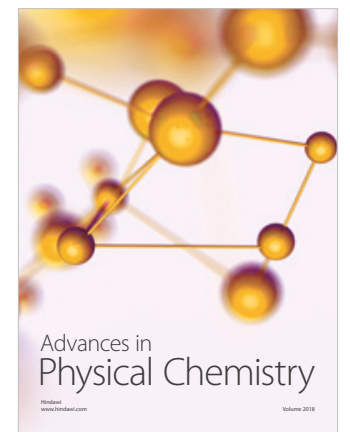

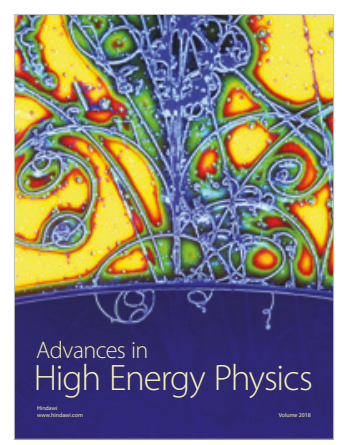

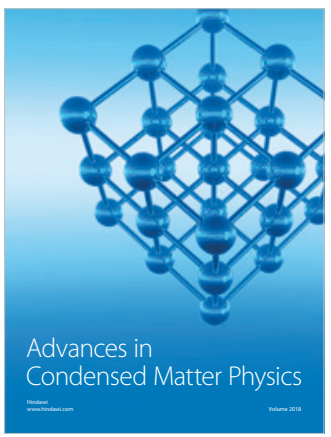

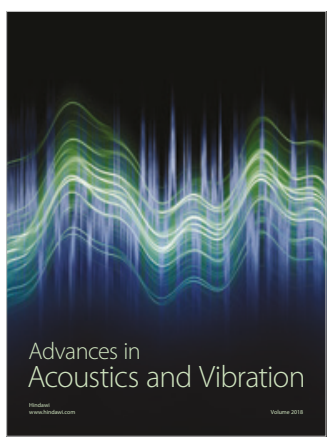

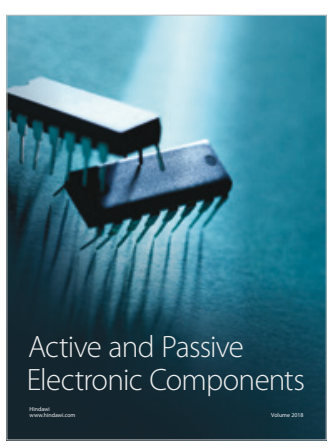
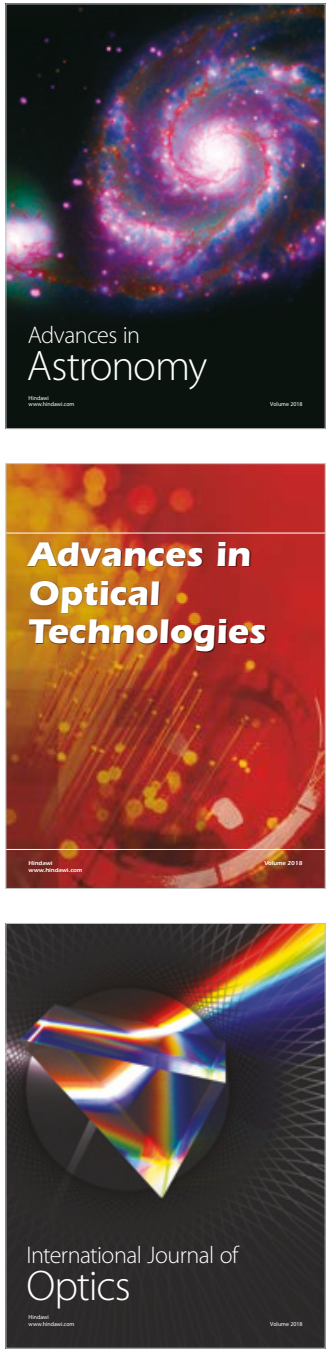